\title{
A Synthetic Spectrum and Light Curve Analysis of the Cataclysmic Variable IX Velorum ${ }^{1}$
}

\author{
Albert P. Linnell ${ }^{2}$, Patrick Godon ${ }^{3}$, Ivan Hubeny ${ }^{4}$, Edward M. Sion $^{5}$, and Paula Szkody ${ }^{6}$ \\ ${ }^{2}$ Department of Astronomy, University of Washington, Box 351580, Seattle, WA \\ 98195-1580 \\ ${ }^{3}$ Department of Astronomy and Astrophysics, Villanova University, Villanova, PA 19085 \\ visiting at the Space Telescope Institute, Baltimore, MD. \\ ${ }^{4}$ Steward Observatory and Department of Astronomy, University of Arizona, Tucson, AZ \\ 85721 \\ ${ }^{5}$ Department of Astronomy and Astrophysics, Villanova University, Villanova, PA 19085 \\ ${ }^{6}$ Department of Astronomy, University of Washington, Box 351580, Seattle, WA \\ 98195-1580 \\ ${ }^{2}$ linnell@astro. washington. edu \\ ${ }^{3}$ godon@stsci.edu \\ ${ }^{4}$ hubeny@as . arizona.edu \\ ${ }^{5}$ edward.sion@villanova.edu \\ ${ }^{6}$ szkody@astro. washington. edu
}

\begin{abstract}
Spectrum synthesis analysis of FUSE and STIS spectra for the cataclysmic variable IX Vel shows that it is possible to achieve a close synthetic spectrum fit with a mass transfer rate of $\dot{M}=5 \times 10^{-9} M_{\odot} / \mathrm{yr}^{-1}$ and a modified standard model temperature profile. The innermost four annuli of the accretion disk model, extending to $r / r_{\mathrm{wd}} \approx 4$, are isothermal; beyond that limit the temperatures follow the standard model.

A light synthesis fit to $\mathrm{K}$ band photometry requires shallow eclipses of the accretion disk rim and secondary star limb. The geometry constrains the orbital inclination to $i=57 \pm 2^{\circ}$. The synthetic light curve requires a vertically extended accretion disk rim, beyond that predicted by gravitational equilibrium, to shadow
\end{abstract}

\footnotetext{
${ }^{1}$ Based on observations made with the NASA/ESA Hubble Space Telescope, obtained at the Space Telescope Science Institute, which is operated by the Association of Universities for Research in Astronomy, Inc. under NASA contract NAS5-26555, and the NASA-CNES-CSA Far Ultraviolet Explorer, which is operated for NASA by the Johns Hopkins University under NASA contract NAS5-32985
} 
the secondary star. The enhanced vertical extension is consistent with recent MHD predictions for CV accretion disks.

Matching differing observed heights of alternate $\mathrm{K}$ band light curve maxima requires a warm rim region downstream of the intersection point of the mass transfer stream with the accretion disk rim. The temperature of the warm region is inconsistent with expectation for a bright spot associated with a rim shock.

Subject headings: Stars:Novae,Cataclysmic Variables,Stars:White Dwarfs,Stars: Individual:Constellation Name: IX Velorum

\section{Introduction}

Cataclysmic variables $(\mathrm{CVs})$ are semi-detached binary stars in which a late main sequence star loses mass onto a white dwarf (WD) by Roche lobe overflow (Warner 1995). In non-magnetic systems the mass transfer stream produces an accretion disk with mass transport inward and angular momentum transport outward driven by viscous processes. The accretion disk may extend inward to the WD; the outer boundary extends to a tidal cutoff limit imposed by the secondary star in the steady state case. If the mass transfer rate is below a certain limit, the accretion disk is unstable and undergoes brightness cycles (outbursts), and if above the limit, the accretion disk is stable against outbursts; the latter case objects are called nova-like (NL) systems.

NL systems are of special interest because they are expected to have an accretion disk radial temperature profile given by an analytic expression (Frank, King \& Raine 1992, eq.5.41)(hereafterFKR) thereby defining the so-called standard model. IX Vel is one such NL system.

IX Vel was discovered as a CV by Garrison et al. (1982) in connection with their MKUBV survey of southern OB stars (Garrison et al. 1977). They reported colors similar to UX UMa, light flickering of order $0.1^{\mathrm{m}}$ on a timescale of minutes, with larger variations on the order of years, and extremely broad, shallow $\mathrm{H}$ absorption lines with emission cores. The light variations in NL systems are thought to arise from variations in the mass transfer rate (Warner 1995, §4.3.1). Wargau et al. (1983) presented additional spectrophotometry, with a radial velocity curve based on mean values of the first three Balmer lines, together with a preliminary estimate of the orbital period, $0.187^{\mathrm{d}}$. Wargau et al. (1984) presented photometric observations showing that the photometric magnitude varied erratically between $m_{p g}=9^{\mathrm{m}} .1$ and $10^{\mathrm{m}} .0$ between Dec. 1963 and Jan. 1973. Their IR photometry showed no obvious eclipse effects, setting a limit to the inclination of roughly $65-70^{\circ}$. Garrison et al. 
(1984) provided further optical spectral data, confirmed that IX Vel is subject to flickering on a time scale of minutes, and derived an estimated mass transfer rate of $7 \times 10^{-10} M_{\odot} \mathrm{yr}^{-1}$. Eggen \& Niemela (1984) confirmed the photometric variation and suspected doubling in the $\mathrm{H} \alpha$ and $\mathrm{H} \beta$ emission lines. Warner, O'Donoghue \& Allen (1985) used high speed photometry to show that IX Vel is variable at the $0.001^{\mathrm{m}}$ level with a non-constant flickering period $\sim 25^{\mathrm{s}}$.

Sion (1985) was first to model the system; he used IUE spectra and called attention to the presence of a wind from the system. Sion showed that the energy distribution is consistent with a mass transfer rate between $10^{-8}$ and $10^{-9} M_{\odot} \mathrm{yr}^{-1}$; his analysis showed that IX Vel is a NL system.

Haug (1988) obtained JHK photometry of the system and showed that there is a lowamplitude IR light curve with an amplitude of a few times 0.01 , with a deeper minimum at phase 0.0 and slightly unequal successive light maxima.

Beuermann \& Thomas (1990) (hereafter BT) detected narrow optical H and He emission lines from the secondary star in IX Vel. The narrow lines were in antiphase to somewhat broader emission lines that varied in phase with the WD and accretion disk, and in turn were superimposed on broad absorption troughs. Radial velocities for the emission components, and a more accurate orbital period $\left(0.1979292^{\mathrm{d}}\right)$, determined component masses for the system $\left(M_{1}=0.80 M_{\odot}, M_{2}=0.52 M_{\odot}\right)$ and a value for the orbital inclination $\left(i=60^{\circ}\right)$. The model also determined a mass transfer rate of $7.9 \times 10^{-9} M_{\odot} / \mathrm{yr}^{-1}$ and a distance of 95 pc. by Bailey's method (see BT for details). BT attributed the emission line excitation to irradiation by the primary or the accretion disk, supporting a suggestion by Hessman (1989). The BT model has been the accepted model to the present time.

Long et al. (1994), hereafter L94, used HUT to obtain a spectrum of IX Vel in the

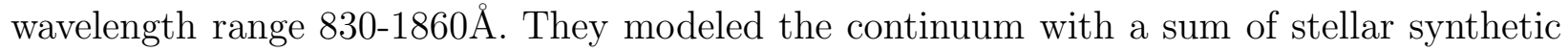
spectra to represent the individual annuli, and assumed that the resulting disk spectrum was the only contributor to the system synthetic spectrum. L94 obtained the important results that: (1) a standard model accretion disk (FKR) does not provide an accurate fit to the HUT spectrum, and (2) the quality of fit is sensitive to the adopted interstellar reddening of the observed spectrum; they state that the WD in the system likely has a $T_{\text {eff }}$ in the range 50,000-75,000K. By contrast, Shaviv \& Wehrse (1991) had found a good standard model fit to spectral energy distribution (SED) data listed by Haug (1987).

Mauche (1991) used high resolution IUE spectra to study the wind associated with IX Vel. Further studies on this topic have been by Prinja \& Rosen (1995) and Hartley et al. (2002).

Kubiak. Poimanski \& Krzeminski (1999) presented phase-resolved spectroscopy which 
provides evidence for an accretion disk rim bright spot contribution to the system spectrum. Polidan. Mauche, \& Wade (1990) detected no extreme-UV flux from IX Vel with Voyager, but van Teeseling et al. (1995) detected X-rays from IX Vel with ROSAT. The latter authors studied ROSAT and EUVE observations of IX Vel and showed that there is an orbital modulation of X-ray flux which probably excludes a boundary layer origin but might arise from a rim bright spot. They also found that the x-ray flux is more than a thousand times smaller than the far UV flux, and that the x-ray flux can be modeled with a single optically thin spectrum.

A motivation for this study is that the FUSE spectra embrace the peak of the IX Vel SED near $1050 \AA$, providing an improved constraint on a synthetic spectrum fit. In addition, the Hipparcos parallax (Perryman et al. 1997) places IX Vel within the Sun's immediate neighborhood and permits an absolute calibration of the mass transfer rate from the secondary star.

\section{Interstellar reddening}

The L94 result emphasizing the importance of interstellar reddening leads us to consider this topic with care.

Garrison et al. (1984) propose $\mathrm{E}(\mathrm{B}-\mathrm{V})=0.03$ for IX Vel and a distance of approximately 100 pc, while Böhnhardt et al. (1982) list $\mathrm{E}(\mathrm{B}-\mathrm{V})=0.02$ based on ESO IUE spectra; by contrast la Dous (1991) lists $\mathrm{E}(\mathrm{B}-\mathrm{V})=0.0$, based on $I U E$ spectra SWP18578 and LWR14655, while Bruch \& Engel (1994) list $\mathrm{E}(\mathrm{B}-\mathrm{V})=0.01$. Mauche et al. (1988) studied narrow interstellar absorption lines on $I U E$ spectra to determine H I column densities near the Sun, determined a value of $20 \times 10^{19} \mathrm{~cm}^{-2}$ in the direction of IX Vel, and note that Verbunt (1987), determined a rough upper limit of $\mathrm{E}(\mathrm{B}-\mathrm{V})$ of 0.04 (and a preferred value of $\mathrm{E}(\mathrm{B}-\mathrm{V})=0.0$ ) based on a study of the $2200 \AA$ "bump" in IUE spectra. L94 note that, if the gas to dust ratio along the line of sight to IX Vel is typical, the reddening corresponding to the column density of H I inferred by Mauche et al. (1988) implies $\mathrm{E}(\mathrm{B}-\mathrm{V}) \approx 0.004 \mathrm{mag}$. L94 found that the best-fitting model spectrum depends on both the adopted $\mathrm{E}(\mathrm{B}-\mathrm{V})$ and the adopted mass transfer rate from the secondary star. With the distance to IX Vel set at 95 pc., from BT, the best-fitting model spectrum corresponded to $\mathrm{E}(\mathrm{B}-\mathrm{V})=0.07$ and a mass transfer rate of $1.3 \times 10^{-8} M_{\odot} / \mathrm{yr}^{-1}$. This mass transfer rate was more than 10 times higher than for the bestfitting model spectrum if $\mathrm{E}(\mathrm{B}-\mathrm{V})=0.0$. L94 found that, on adopting the mass transfer rate derived by $\mathrm{BT}$, the best-fitting model spectrum corresponded to $\mathrm{E}(\mathrm{B}-\mathrm{V})=0.03$. Based on the available data we believe the most likely $\mathrm{E}(\mathrm{B}-\mathrm{V})=0.01$, which we adopt and confirm á postiori. 


\section{The FUSE and $S T I S$ spectra}

The observations log is presented in Table 1 .

\subsection{The FUSE Spectrum}

The spectrum of IX Vel was obtained through the 30"x30" LWRS Large Square Aperture, in HISTOGRAM mode. The data were processed with CalFUSE version 3.0.7. The main change from previous versions of CalFUSE is that now the data are maintained as a photon list (the intermediate data file - IDF) throughout the pipeline. Bad photons are flagged but not discarded, so the user can examine the filter, and combine data without rerunning the pipeline. The spectrum actually consists of 12 exposures of about 600 sec each, and these exposures, totaling $7336 \mathrm{sec}$ of good exposure time, were combined (using the IDF file). Consequently, the FUSE spectrum has a duration of about 0.44 of the period of IX Vel. The spectral regions covered by the FUSE spectral channels overlap, and these overlap regions are then used to renormalize the spectra in the $\mathrm{SiC} 1, \mathrm{LiF} 2$, and $\mathrm{SiC} 2$ channels to the flux in the LiF1 channel. We combined the individual channels to create a time-averaged spectrum with a linear $0.1 \AA$ dispersion, weighting the flux in each output datum by the exposure time and sensitivity of the input exposure and channel of origin. In this manner we produced a final spectrum that covers the full FUSE wavelength range $905-1187 \AA$.

\subsection{The HST/STIS spectrum}

The STIS spectrum was processed with CALSTIS version 2.22. The HST/STIS spectrum of IX Vel was obtained in TIME TAG operation mode using the FUV MAMA detector. The observations were made through the $0.1 \times 0.03$ aperture with the E140M optical element. The STIS spectrum has a duration of $1750 \mathrm{sec}$ or about 0.1 the period of IX Vel. The STIS spectrum, centered on the wavelength $1425 \AA$, consists of 42 echelle spectra that we extracted and combined to form a single spectrum from about $1160 \AA$ to $1710 \AA$. At very

short wavelengths the STIS spectrum is very noisy and we disregard the region $\lambda<1160$ $\AA$; towards longer wavelengths the echelle spectra do not overlap and five gaps are apparent around $\lambda \approx 1634 \AA, 1653 \AA, 1672 \AA, 1691 \AA$, and $1710 \AA$.

Figure 1 shows the FUSE and the STIS spectra of IX Vel. 


\subsection{The Lines}

The FUV spectra of accreting WDs in CVs exhibit absorption and emission lines from the WD, the accretion disk, the hot component (possibly the inner disk or boundary/spread layer) as well as sharp absorption lines from circumbinary material and/or the ISM. Additionally, terrestrial contamination is also usually observed, especially in FUSE spectra.

The main characteristic of the FUSE spectra of CVs is the broad Ly $\beta$ absorption feature due to the large gravity $(\log g \approx 8)$ and temperature of the WD, while the Ly $\alpha$ absorption feature is observed in the STIS spectral range. In the disk these features are usually broader and smoothed out unless the inclination of the system is very low and the disk is almost face-on. At higher temperatures, as the continuum rises in the shorter wavelengths, the higher orders of the Lyman series also become visible in the FUSE range.

Examination of the FUSE spectrum reveals that the continuum is very slightly affected by deep and sharp ISM H I absorption towards shorter wavelengths, extending to the hydrogen cut-off around $915 \AA$. We annotated the position of these hydrogen lines under the first panel of Figure 1. Other lines which are usually attributed to the ISM and/or to air contamination are the O I lines and the low order Nitrogen lines N I \& N II. All these lines are observed in the FUSE spectrum of IX Vel. Since these lines are sharp and are observed at their theoretical wavelengths they are not associated with the source, though they could be from circumbinary material ejected from the system. Of particular interest are the Si II, S II, and C II lines observed in the STIS spectral range. These lines are usually observed as broad absorption lines originating from the WD atmosphere. Here, these lines are sharp, and, like the Hi, O I, N I, and N II lines, they are located at their theoretical wavelengths. This seems to indicate that the circumbinary material in the line of sight was ejected from the WD/accretion disk. The sharp Si III $1206.5 \AA$ line is at its theoretical wavelength and appears to be circumbinary. Not as obvious, but also apparently coming from the circumstellar gas, are the sharp lines of Si IV, and C IV superposed on the broad absorption lines.

All the broad absorption lines in the FUSE spectrum (N IV, S vi, C III, N III, O vi, H I, S IV ,...) and in the STIS spectrum (C III, Si N V, Si IV, and C IV) have the same shape with a sharp steep red wing and a much broader blue wing. The H I, C IV, and He II lines do have a pronounced P Cygni profile while all the remaining broad absorption lines present only a hint of possible red shifted emission. This is an indication that the FUSE and STIS spectra were obtained at about the same orbital phase (see e.g. Hartley et al. 2002).

In Table 2 and Table 3 we identify all the absorption lines of the FUSE and STIS spectra. The wavelength tabulations are the theoretical wavelengths. 


\subsection{Combining the spectra}

We consulted the AAVSO (American Association of Variable Stars Observers) light curve generator to check the magnitude of IX Vel at the time of the observations. However, the AAVSO data did not provide data on the specific days the observations were made but a few days before and later. The source appeared to vary erratically between about magnitude

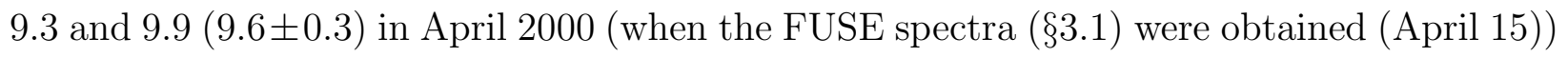
and between about magnitude 9.5 and $10.1(9.8 \pm 0.3)$ in July of the same year (when the STIS spectrum was obtained (July 19)). (Hartley et al. (2002), their Figure 1 and Table 2, assign this observation to August 19 instead of July 19.) To within the 0.3 magnitude uncertainty, the FUSE spectrum and the STIS spectrum were at about the same magnitude $(\approx 9.6$ versus $\approx 9.8)$. In addition, from the flux level of the FUSE and STIS spectra it is clear that the system was in the same state and with the same mass accretion rate. Finally, from a visual inspection of the absorption lines it is clear that the spectra were obtained at about the same orbital phase.

Before combining the FUSE and STIS spectra, we stress that (i) the system was in the same state at the two times of observation; (ii) the shapes of the spectra (continuum and lines) are similar (where the spectra are not too noisy); (iii) the flux level in the FUSE and HST/STIS spectra do not differ more than about 5\% (the FUSE and HST/STIS are very different instruments and we do not expect their flux level to match exactly); (iv) the spectra have been obtained at about the same binary phase (this is important since IX Vel is a high inclination system for which the observing time is significantly shorter than the orbital period). We have adopted a divisor of 1.03, applied to the STIS spectrum, in placing the two spectra on the same plot. We have also removed flux entries from both spectra which were either 0.0 or negative.

\section{The analysis program:BINSYN}

Our analysis uses the program suite BINSYN (Linnell \& Hubeny 1996); a recent paper (Linnell et al. 2007) describes its application to a CV system in detail. Briefly, an initial calculation produces a set of annulus models for a given WD mass, radius, and mass transfer

rate. This calculation uses the program TLUSTY (Hubeny 1988; Hubeny \& Lanz 1995). The program considers the radial and vertical structure of the disk independently. The radial structure is based on the standard model (FKR) and so follows the prescribed relation between local $T_{\text {eff }}$ and the annulus radius. The vertical structure is solved, self-consistently, as described by Hubeny (1990) and Hubeny \& Hubeny (1998). The set of annulus models covers the accretion disk from its innermost (WD) radius to just short of its outermost (tidal 
cutoff) radius. Our final model, discussed below, adopts an outer accretion disk $T_{\text {eff }}$ which the final and penultimate annulus models bracket.

Table 4 lists properties of annuli calculated with TLUSTY(v.202) for a mass transfer rate of $5.0 \times 10^{-9} M_{\odot} / \mathrm{yr}^{-1}$. The control file to calculate a given annulus requires a radius of the WD in units of $R_{\odot}$. All of the annuli used the radius of a zero temperature WD for a homogeneous carbon Hamada-Salpeter $0.80 M_{\odot}$ model from Panei. Althaus, \& Benvenuto (2000). All of the annuli are H-He models, and the models through $r / r_{\mathrm{wd}, 0}=20.00$ are converged LTE models. The remaining annuli are LTE-grey models (see the TLUSTY Users Guide for an explanation). Each line in the table represents a separate annulus. The column headed by $m_{0}$ is the column mass, in $\mathrm{gm} \mathrm{cm}^{-2}$, above the central plane. The column headed by $T_{0}$ is the upper boundary temperature in degrees Kelvin, with the upper boundary specified as that level with a column mass of $1.0 \times 10^{-5} \mathrm{gm} \mathrm{cm}^{-2}$ lying between it and the next inward tabular level. The entire annulus has a total of 70 levels. Compare with the annulus $T_{\text {eff }}$ value, which references the local temperature at a Rosseland optical depth of $\approx 0.7$. The columns headed by $z_{0}$ and $N_{e}$ are respectively the upper boundary height $(\mathrm{cm})$ above the central plane and the electron density $\left(\mathrm{cm}^{-3}\right)$ at the upper boundary. The $z_{0}$ value in the final row of Table 4 determines the rim semi-height. The column headed by log $g$ is the log gravity in the $z$ direction at a Rosseland optical depth of $\approx 0.7$. The column headed by $\tau_{\text {Ross }}$ is the Rosseland optical depth at the central plane. We call attention to the fact that the annuli are optically thick to the outer radius of the accretion disk.

Following calculation of annulus models for assigned $M_{\mathrm{wd}}$ and $\dot{M}$, program SYNSPEC(v.48) 1 (Hubeny, Lanz, \& Jeffery 1994) is used to produce a synthetic spectrum for each annulus. We adopted solar composition for all synthetic spectra. The synthetic spectra include contributions from the first 30 atomic species, assumed to be in LTE.

SYNSPEC optionally produces either flux values or intensities at a specified number of zenith angles for the individual annulus synthetic spectra. The spectra, produced in BINSYN by interpolation among the SYNSPEC spectra, require intensity data for integration over the visible object. If the SYNSPEC output is in flux units, BINSYN associates a limb darkening value to produce intensity values at required directions. We tested both the intensity and flux SYNSPEC options for the simulations described below, using a limb darkening value of 0.6 in connection with the flux output; the difference between the two types of system synthetic spectra was nearly undetectable.

BINSYN models the complete CV system, including the WD, secondary star, accretion disk face, and accretion disk rim as separate entities. The model represents phase-dependent

\footnotetext{
${ }^{1}$ http://nova.astro.umd.edu
} 
and inclination-dependent effects, including eclipse effects and irradiation effects, on all of the system objects. The representation of the stars requires polar $T_{\text {eff }}$ values and gravitydarkening exponents. BINSYN represents the accretion disk by a specified number of annuli, where that number typically is larger than the number of TLUSTY annulus models. The accretion disk $T_{\text {eff }}$ profile may follow the standard model, but, alternatively, the profile may be specified by a separate input file. The contributions of the individual BINSYN annuli to the BINSYN representation of the accretion disk face follows by interpolation within the set of SYNSPEC spectra for the TLUSTY annuli. The $T_{\text {eff }}$ of the accretion disk rim ties to the outermost annulus $T_{\text {eff }}$ via the theory by Hubeny \& Plaved (1991).

BINSYN has provision to represent a bright spot on the rim face, with specified local $T_{\text {eff }}$, angular extent, and $T_{\text {eff }}$ taper, upstream and downstream, relative to the specified bright spot location. The output synthetic spectra include the separate contributions of the two stars, the accretion disk face, the accretion disk rim, and the composite system spectrum.

BINSYN also can calculate a synthetic light curve for specified wavelength (see $§ 6.2$ and the following sections).

\section{System parameters adopted initially}

Initially adopted parameters from BT (Table 1) included $M_{\mathrm{wd}}=0.80 M_{\odot}, M_{\mathrm{sec}}=$ $0.52 M_{\odot}, \mathrm{P}=0.1939292^{\mathrm{d}}$, and $i=60^{\circ}$. Panei, Althaus, \& Benvenuto (2000) lists a WD radius of $9.8459 \times 10^{-3} R_{\odot}$ for a $0.80 M_{\odot}$ homogeneous zero temperature Hamada-Salpeter carbon model WD. In our subsequent study of the observed spectra we correct the radius for the adopted WD $T_{\text {eff }}$; the contribution of the WD to the system synthetic spectrum is appreciable. In the immediately following study of the $\mathrm{K}$ band light curve the relatively insignificant and constant contribution of the WD is absorbed in the normalization of system light to its maximum value. Consequently, we use the accretion disk as calculated for the zero temperature WD in the light curve study. The secondary star mass and the results of Kolb \& Baraffe (2000) determine a secondary star spectral type of M2. This spectral type and Table 15.7 of Cox (2000) determines a polar $T_{\text {eff }}$ of $3500 \mathrm{~K}$ for the secondary star. This is the same temperature adopted by BT (see our Table 5). Note that a main sequence class V star of the same mass would have a spectral type slightly earlier than M0 (Table 15.8 of Cox (2000) ) and a polar $T_{\text {eff }}$ of $3900 \mathrm{~K}$. In calculating irradiation of the secondary star we have adopted a bolometric albedo of $A_{\mathrm{s}}=0.6$ (Claret 2001); $A_{\mathrm{wd}}=1.0$. We have used standard values for gravity darkening exponents of $\beta_{\mathrm{wd}}=0.25$ and $\beta_{\mathrm{s}}=0.08$.

A number of studies have considered the tidal cutoff boundary, $r_{d}$, of accretion disks 
(Paczynski (1977); Papaloizou \& Pringle (1977); Whitehurst (1988); Schwarzenberg-Czerny \& Różyczka (1988); Whitehurst \& King (1991); Goodman (1993)). These authors agree on $r_{d} \approx 0.33 D$, where $D$ is the separation of the stellar components. We adopt this expression for the tidal cutoff radius of the accretion disk.

The Hipparcos parallax (Perryman et al. 1997) is $10.38 \pm 0.98$ mas, corresponding to a distance of $96.3 \mathrm{pc}$ which we adopt for the system distance.

\section{An IX Velorum system model}

\subsection{An initial model}

Starting with the parameters of the previous section, we added an explicit model of the accretion disk. Using TLUSTY annulus models for $M_{\mathrm{wd}}=0.80 M_{\odot}$ (still zero temperature) and a mass transfer rate of $8.0 \times 10^{-9} M_{\odot} / \mathrm{yr}^{-1}(\mathrm{BT})$, we calculated a standard model system synthetic spectrum. The rim semi-height, $0.085 R_{\odot}$, followed from the TLUSTY model for the outer annulus, and the radius of the accretion disk was $0.56 R_{\odot}$, the tidal cutoff radius. We divided the synthetic spectrum by $8.8299 \times 10^{40}$, the scaling factor corresponding to the Hipparcos distance of $96.3 \mathrm{pc}$, for comparison with the unreddened observed spectrum. The synthetic spectrum flux was appreciably too high over the entire spectral range of the FUSE plus STIS spectra for our adopted reddening of $\mathrm{E}(\mathrm{B}-\mathrm{V})=0.01$. A standard model synthetic spectrum with $\dot{M}=5.0 \times 10^{-9} M_{\odot} / \mathrm{yr}^{-1}$ had a much improved overall fit. In this model the rim semi-height is $0.080 R_{\odot}$, equal to the height of the outermost TLUSTY (equilibrium) model. Although the synthetic spectrum was slightly too blue, and the flux level too high, we anticipated improving both the spectral gradient and the flux level by modifying the accretion disk temperature profile from the standard model.

The inclination $i=60^{\circ}(\mathrm{BT})$ is a maximum value, for a test model, to avoid deep eclipses which are not observed. In fact, this model has grazing eclipses; we tested the effect on the synthetic spectrum of reducing $i$ to $54^{\circ}$, which barely avoids eclipses for the current model. Because of the reduced inclination, flux from the $54^{\circ}$ model was both too large and too blue. Reducing the mass transfer rate has a very small effect on the continuum gradient, so we modified the accretion disk temperature profile. The hot inner annuli have the strongest effect on the spectral gradient. We reduced the temperatures of the innermost four annuli to $30,000 \mathrm{~K}$ and produced a fairly close fit to the observed spectral gradient and flux level, still at $i=54^{\circ}$. The outer annuli make small contributions to the synthetic spectrum, so the synthetic spectrum is relatively insensitive to changes in the temperature profile in the outer accretion disk. However, the light curve results are sensitive to the temperature structure of 
the outer accretion disk. Consequently, at this stage we calculated a $\mathrm{K}$ band $(2.2 \mu)$ synthetic light curve for comparison with the observational data by Haug (1988).

\subsection{Treatment of the disk rim and a $\mathrm{K}$ band synthetic light curve}

We used the Dexter facility of the Astrophysics Data System (hereafter ADS) to extract the observed points from Haug's Fig.7. Our program PGCAC, within the BINSYN suite, evaluates irradiation of the secondary star due to the WD, the accretion disk faces, and the accretion disk rim. The effects are calculated separately, permitting an evaluation of the independent effects. The combined effects, with the adopted rim semi-height of $0.080 R_{\odot}$, produced an Algol type light curve, with a single light maximum per orbital cycle near phase 0.5 , and in strong conflict with the observations. This situation had been anticipated by BT, and they had proposed an irradiation model in which the rim height is high enough to shield most of the secondary from the WD.

This new constraint requires replacing the annulus heights calculated by TLUSTY; it also means that the previously calculated synthetic spectra must be recalculated. We set the semi-height of the rim to an empirical (i.e., not theoretically-based) and iterativelydetermined height of $0.20 R_{\odot}$; this height barely shadows the poles of the secondary from the WD, but unavoidably guarantees that shallow eclipses of the secondary star and the accretion disk take place, even for an inclination of $54^{\circ}$. The secondary star is partially irradiated by the accretion disk face regions more remote than the WD. We discuss the light curve analysis below, but with the system geometry now approximately fixed we first focus on production of a suitable synthetic spectrum. We found that very good fits to the STIS + FUSE spectra are possible by adjusting the $T_{\text {eff }}$ values of the four innermost annuli, with $i$ between $54^{\circ}$ and $60^{\circ}$, the $\dot{M}=5.0 \times 10^{-9} M_{\odot} / \mathrm{yr}^{-1}$ temperature profile for the remainder of the accretion disk, and reddening corresponding to $E(B-V)=0.01$. Figure 2 portrays the system projected on the plane of the sky at orbital phase 0.0 , for an orbital inclination of $57^{\circ}$.

The following text describes a further adjustment of the accretion disk temperature

profile, but it is first necessary to return to the light curve analysis. Haug (1988) attempted a simulation of the light curve based on the assumption that the secondary star and the WD both have $T_{\text {eff }}=3550 \mathrm{~K}($ !), that irradiation of the secondary is by an accretion disk with $T_{\text {eff }} \leq 5100 \mathrm{~K}(!)$, and that the bolometric albedo of the secondary is 1.0 . There was no evaluation of shadowing effects nor details concerning the irradiation calculation. Haug obtained a difference in depths of the minima by setting the secondary star bolometric albedo to 1.0. Although the calculated light curve fitted the observations fairly well, the Haug parameters are grossly unrealistic. Haug was unable to simulate the difference in the 
alternate light maxima.

Shadowing of the secondary by the increased rim height has an important effect on the temperature profile of the secondary star. In the absence of any irradiation, the $T_{\text {eff, }}$ of the secondary star photospheric point, essentially coincident with the L1 point (hereafter 'point'), is $618 \mathrm{~K}$ in our model (the calculated point gravity is small but not zero). Although the point receives no irradiation from the $\mathrm{WD}$ or faces of the accretion disk, there is irradiation by the accretion disk rim. This irradiation produces a point $T_{\text {eff }}$ value of $3119 \mathrm{~K}$. Irradiation of the secondary star by the rim affects the calculated light curve.

The light loss at the successive minima is a complex function of the temperature distribution on the object undergoing eclipse and the eclipsed areas. Only a segment of the accretion disk rim undergoes eclipse, and the rim temperature depends strongly on the system model. In our model the top of the rim barely shadows the poles of the secondary star from the WD. BINSYN does not have the capability to simulate semi-transparent regions, but we assume the top of the rim permits passage of enough photons to produce the observed secondary component emission lines, originating near the secondary star poles. We use the theory of Hubeny \& Plaved (1991) to relate the rim temperature to the temperature of the outermost annulus, which the standard model assumes to be in Keplerian motion. Briefly, the outer region of the accretion disk is not expected to be precisely in Keplerian motion, the outer boundary is expected to be approximately semi-toroidal in cross-section and to have an effective temperature given by

$$
T_{\text {eff,rim }} / T_{\text {eff,Kep }}=(8 q / 9)^{1 / 4},
$$

where

$$
q=\frac{4}{\pi} \frac{1+(h / 2)}{[1+(2 / \pi) h][1+2 h]}, \quad h=\frac{H}{R}
$$

where $H$ is the semi-height of the rim and $R$ is the radius of the rim upper edge, measured in the central plane. The temperature of the outermost annulus depends on the mass transfer rate; for the adopted $\dot{M}$, and the standard model, that temperature is $5659 \mathrm{~K}$, giving $T_{\text {eff,rim }}=$ $5052 \mathrm{~K}$. That temperature is below the $T_{\text {eff }}$ of the outermost annulus in Table 4 because the rim radius is slightly larger than the radius of the last calculated annulus. Note that the temperature in question is below the temperature nominally adopted for transition to the hot state $(\approx 6300 \mathrm{~K}($ Smak 1982) $)$. We defer any discussion of this point and proceed to calculate a $\mathrm{K}$ band light curve for the current system parameters.

For light curve purposes, we represent the radiation from a given area segment on any of the system objects by a black body at the local $T_{\text {eff }}$. The light intensity at polar angle $\gamma$ relative to the local surface normal and at wavelength $\lambda$ is

$$
I_{\lambda}(\gamma)=B_{\lambda}\left[1-u_{1}(1-\cos (\gamma))-u_{2}(1-\cos (\gamma))^{2}\right]
$$


where $B_{\lambda}$ is the black body intensity. The quadratic limb darkening coefficients, $u_{1}$ and $u_{2}$, are from Wade \& Rucinski (1985). Before presenting the light curve calculations it is necessary to consider the different heights of the alternate light maxima.

\subsection{A bright spot}

Kubiak. Poimanski \& Krzeminski (1999) use their artificial spectrum-template generator (Pojmański \& Udalski 1997) to produce phase-resolved artificial spectra for comparison with observed spectra. Their three-component model, with one component a bright spot, is a close match to the summed observed spectrum. They derive an orbital phase of 0.75 for the bright spot. With respect to the observed light curve, we assume the difference in successive maximum light levels arises from the interaction of the transfer stream and the accretion disk outer boundary region. Figure 3 shows a plan view of the system. The mass transfer stream follows the theory of Lubow \& Shu (1975). The stream enters the rim at an angle of $62^{\circ}$ relative to a tangent line at the impact point. If the bright spot were centered at the impact point the higher light maximum would occur at orbital phase 0.90, contrary to observation. A warm downstream rim region centered at orbital phase 0.75 , as derived by Kubiak, Pojmanski \& Krzeminski (1999), produces a good fit to the observed light maxima.

This location is close to the orbital phase 0.8 found in other systems (Szkody 1992; Livio 1993). We assume the location and $T_{\text {eff }}$ of the warm rim region roughly represents that dissipation signature. Figure 4 shows the system at orbital phase 0.75 ; the dark rim area indicates the warm region. In this figure the secondary star is approaching the observer and the warm region is at its maximum phasewise exposure. The light curve is not strongly sensitive to the phasewise location of the spot; variation of 0.05 produces little effect on the calculated light curve. Our software cannot produce a bright spot more restricted in height on the rim than shown. The dark rim area, Figure 4, does not represent the tapered temperature profile at the spot beginning and end. A spot model with an oval appearance would produce a photometric signature differing negligibly from the model presented here.

\subsection{Synthetic light curve fits and implications}

Figure 5 presents the synthetic $\mathrm{K}$ band light curve overplotted on the photometric observations. The observations (Haug (1988)) are for 1984 January 16-23 (crosses) and 1987 January 12-16 (asterisks). The plotted points represent observations binned in 0.1 phase intervals, with initial numerical values measured relative to the mean for the time interval in question. The original Haug observations are in differential magnitudes. Haug estimates 
the individual observations have a 0.03 mag.accuracy. The binned observations are more accurate than this. The change in the light curve between the two dates is believed to be real. We have applied a -0.025 magnitude correction to the observations for optimum fit to the normalized theoretical light curve. Note that the ordinate is in flux units, not magnitudes.

The depths of the light minima are functions of the orbital inclination; it is of interest to determine the sensitivity of the minima to the inclination. Figure 6 shows the synthetic light curve for $i=54^{\circ}$, and Figure 7 is the plot for $i=60^{\circ}$. All other parameters have remained fixed. Based on the three plots, we argue that the best fit is for $i=57^{\circ}$ and that the estimated accuracy is $\pm 2^{\circ}$. The light curve geometric requirement for a shallow eclipse of the accretion disk rim also confirms the tidal cutoff radius of the accretion disk used in this analysis.

The observations for the two epochs show differences that may reflect bright spot changes arising from changes in the mass transfer rate. For this reason it is inadvisable to attempt an optimized light curve fit.

We call attention to the greater primary minimum phase width than presented by the Figure 5 model, for both sets of observations. The "break" in the synthetic light curve on both the ingress and egress phases coincides with the beginning and end of geometric eclipse of the accretion disk rim. The observed light reduction prior to that geometric eclipse indicates that eclipse of a light source already is in progress. It is tempting to associate the light reduction with eclipse of a light source associated with the wind known to be present (and discussed below). Actual demonstration of this speculation as a physical possibility would require extended analysis beyond the scope of this paper. As can be seen from Figure 2, the limb of the secondary star would start to eclipse a wind region before beginning eclipse of the accretion disk rim. Inclusion of an additional light source (similar to "third light" in classical binary systems) would require renormalization of the system light unit and so would reduce the calculated depth of the phase 0.0 light minimum, improving the synthetic light curve fit to the observations.

\subsection{The $\operatorname{rim} T_{\text {eff }}$ and the final system model}

The $T_{\text {eff }}$ values of the outer annuli in the current light curve model are below the transition value to the hot state, which has a minimum $T_{\text {eff }}$ of approximately $6300 \mathrm{~K}$ (Smak 1982). That value assumes the accretion disk is thin and that hydrostatic equilibrium results from gravitational acceleration only. To make sure our model is free of instability (Osaki 
1996) we adopt a model for which those annuli whose standard model $T_{\text {eff }}$ values fall below $6300 \mathrm{~K}$ are reset to $6300 \mathrm{~K}$. We have correspondingly revised the parameters of the warm spot region; the new rim model parameters are in Table 6. For an outer annulus temperature of $6300 \mathrm{~K}$, equations (1), (2) lead to a corresponding rim temperature of $5624 \mathrm{~K}$ outside the warm spot region. Irradiation of the rim by the secondary star raises the rim temperature at the line of (stellar) centers intersection to $5628 \mathrm{~K}$. The temperature profile for the light curve model and the corresponding standard model are in Table 7.

The 'transition temperature' between the hot and cool states falls within a range of values (Smak 1982). Our light curve model avoids the instability issue by adjusting the temperature of the outer accretion disk to be at the low end transition temperature, 6300K. Our rim model obviously is a somewhat rough approximation; in view of the uncertainties associated with the vertical extent of the rim we believe the approximation is appropriate.

Figure 8 shows the comparison of the new synthetic light curve and the photometric observations. The fit is nearly indistinguishable from that of Figure 5. Although the light curve fits do not distinguish between the standard model and the model with warmer outer annuli, there are theoretical reasons favoring the latter model. The standard model does not account for expected warming of the outer accretion disk region when the transfer stream shock is hidden at a large optical depth (Pringle 1977). The standard model also does not account for tidal dissipation in the outer accretion disk (Lasota 2001).

We now perform a new simulation of the observed spectra including an allowance for the effect of photospheric temperature on the WD radius. Based on L94, we adopted a $T_{\text {eff }}$ of $60,000 \mathrm{~K}$ for the WD. Using Panei, Althaus, \& Benvenuto (2000, Figure 4a), we determined a radius of $1.50 \times 10^{-2} R_{\odot}$ for the $60,000 \mathrm{~K} \mathrm{WD}$. The WD contribution to the system synthetic spectrum is larger than with the zero temperature WD. We calculated a new BINSYN accretion disk model based on the revised WD radius. This model required a 29,000K isothermal region, as compared with the $30,000 \mathrm{~K}$ isothermal region for the zero temperature WD, for an optimum fit to the observed spectra. A tabulation of the new model temperature profile and the corresponding standard model, is in Table 8. Compare with Table 7. The new model has a slightly different profile with the entire accretion disk on the hot branch. Note that the outer annulus temperature is identical with the corresponding adopted temperature in Table 7. The new model represents our final model. Parameters of the final model are in Table 9. 


\subsection{Confrontation of final model and observational data}

Figure 9 compares the system synthetic spectrum with the combined STIS and FUSE spectra. The contribution of a $60,000 \mathrm{~K}$ WD is at the bottom. The STIS spectrum is the heavier gray plot; the FUSE spectrum is the lighter one. Except near Ly $\alpha$ and the short wavelength end of the FUSE spectrum the fit is very good, allowing for the unmodeled wind lines. Based on the line wings, Ly $\alpha$ appears to be in emission, with a central reversal and a superposed narrow wind line. The strong N V wind line at $1237 \AA$ depresses what otherwise apparently would be a redward emission wing of Ly $\alpha$, symmetric with the blueward emission wing. Figure 10 shows a detail of Figure 9.

SYNSPEC optionally permits production of a continuum spectrum plus lines of $\mathrm{H}$ I and He II. The importance of line opacity of other atomic species is illustrated by Figure 11, which overplots the continuum spectrum for the accretion disk plus rim plus line spectrum of the WD on the same observed spectra as Figure 9, and with the same scaling factor.

We stress that the divisor, $8.8299 \times 10^{40}$, applied to the synthetic spectrum in placing it in Figure 9 is the appropriate scaling factor if the Hipparcos distance to IX Vel is accurate. It was not determined as an optimum divisor to produce an empirical fit to the observed spectra; the scaled synthetic spectrum represents an absolute flux calibration for comparison with the observed, reddening corrected, spectra. The reddening correction, $E(B-V)=0.01$, was assigned prior to and independently of the Figure 9 plot.

Different divisors appropriate to the $\pm 1 \sigma$ uncertainty in the Hipparcos parallax produce synthetic spectrum offsets from the observed spectra that are approximately 1 dex larger than the Figure 9 residuals. The very good synthetic spectrum fit was achieved by first adjusting the mass transfer rate and finally by modifying the accretion disk temperature profile. The temperatures at the first five division radii, Table 8, are critically important. (The flux contribution for a given annulus specified within BINSYN is calculated using the temperature at its inner edge, as given in Table 8.) Higher temperatures, appropriate to the standard model for the adopted mass transfer rate, not only make the accretion disk too luminous and too blue, they raise the continuum shortward of Ly $\alpha$ and appreciably reduce the model depths of the Lyman series absorption lines. We caution that, since IX Vel varies by up to $0.9 m_{p g}$ (Wargau et al. 1984), a suitable model at a different epoch could require a different $\dot{M}$ and a different accretion disk temperature profile.

Figure 12 presents the synthetic continuum spectrum flux over a wide wavelength range, together with the contributions of the separate objects in the system. The triangles represent

flux equivalents of the UBVRI photometry by Garrison et al. (1984); the squares represent flux equivalents of JHKL photometry by Wargau et al. (1984) and Haug (1988). Figure 13 
presents a detail of Figure 12, showing a portion of the Balmer series. The gray line is an optical spectrum by Wargau et al. (1983). We used the curve-following feature of the ADS Dexter facility to extract the plot from the original publication. Note that the observed spectrum shows narrow emission of $\mathrm{H} \beta$ and $\mathrm{H} \gamma$ superposed on shallow absorption features. In producing the flux equivalents we have used the flux calibrations in Table 1 of Holberg \& Bergeron (2006) for the $U, B, V, R, I, J, H, K$ bands and the table on p.202 of Allen (1973) for the L band.

Figure 14 shows a line spectrum corresponding to Figure 13. For clarity, all synthetic spectra are continuous lines. As is true in the cases of Figure 9 and Figure 11, the line spectrum falls below the continuum spectrum and, in the present instance, the Figure 13 and Figure 14 synthetic spectra bracket the observed data.

The secondary star spectrum contributes strongly to the system synthetic spectrum longward of $3000 \AA$. It could be argued that a higher $T_{\text {eff }}$ for the secondary star would improve the fit. We have used a coarse representation of the secondary star: a single $3500 \mathrm{~K} \log g=4.0$ synthetic spectrum calculated with SYNSPEC from a Kurucz main sequence model. A more accurate secondary star representation would require a much more sophisticated synthetic spectrum, calculated from a grid of accurate source synthetic spectra covering appropriate ranges of $T_{\text {eff }}$ and $\log g$ and properly simulating the variation of both $T_{\text {eff }}$ and $\log g$ over the secondary star photosphere. Howell \& Skidmore (2000) caution that secondary stars may be poorly represented by main sequence models. Given the uncertainties in the BT parameters we consider the model fit to the observed data, Figure 12, to be entirely satisfactory.

In view of the dependence of synthetic spectrum fit quality on the adopted $E(B-V)$ studied by L94, we have plotted our final model against the IX Vel observed spectra for E(B$\mathrm{V})=0.0$ (i.e., the actual observed spectra without a reddening correction), for comparison with our adopted $\mathrm{E}(\mathrm{B}-\mathrm{V})=0.01$. In that case our final model flux is too large; we produced an optimum fit with a normalizing divisor of $1.00 \times 10^{41}$, corresponding to an IX Vel distance of $91 \mathrm{pc}$, slightly closer than one standard deviation from the Hipparcos distance. The fit quality is close to that of Figure 9. We also plotted our final model against the IX Vel observed spectra for $\mathrm{E}(\mathrm{B}-\mathrm{V})=0.04$. The optimum fit required a normalizing divisor of $6.10 \times 10^{40}$, corresponding to a distance of $112 \mathrm{pc}$, slightly farther than one standard deviation beyond the Hipparcos distance. The synthetic spectrum had a spectral gradient that was too small. We believe these comparisons support our choice of $\mathrm{E}(\mathrm{B}-\mathrm{V})=0.01(\S 2)$. 


\section{Discussion}

\subsection{Constraints on $\dot{M}$ from orbital inclination and accretion disk $T_{\text {eff }}$ profile}

The geometric constraints from a fit to the $\mathrm{K}$ band photometry are of particular importance. The initial constraint on orbital inclination, $60 \pm 5^{\circ}$, determined from emission line light curves of $\mathrm{H} \alpha$ and $\mathrm{H} \beta$ (BT), limits permissible values of $\dot{M}$ by comparison with the FUSE and STIS spectra because of the dependence of system flux on orbital inclination. In particular, a value $\dot{M}=8 \times 10^{-9} M_{\odot} / \mathrm{yr}^{-1}$ clearly is too large, and a value $\dot{M}=5 \times 10^{-9} M_{\odot} / \mathrm{yr}^{-1}$ is at the lower limit of associated outer boundary temperature, on the standard model, to avoid accretion disk instability (Smak 1982; Osaki 1996). Adopting the latter $\dot{M}$, a fit to the observed spectra requires modification of the standard model temperature profile; a satisfactory fit can only be achieved by adopting an isothermal inner portion of the accretion disk.

Figure 15 illustrates this point; the plot shows the standard model (unmodified) compared with the two observed spectra (compare with Figure 9, representing the modified standard model). The normalizing divisor is $1.30 \times 10^{41}$; it places IX Vel more than two standard deviations farther away than the Hipparcos distance. The synthetic spectrum Lyman lines are much shallower than in Figure 9. This feature is a result of the very shallow Lyman lines in the higher temperature annulus spectra and is a separate argument supporting our isothermal region substitution for the standard model temperature profile. The standard model synthetic spectrum passes through the tops of the observed spectral lines shortward of $975 \AA$, but does not represent the deep Lyman absorption lines in the observed spectra.

It is of interest that our isothermal region result agrees with tomography of three of six CV systems studied by Rutten, van Paradijs, \& Tinbergen (1992) (SW Sex, LX Ser, V1315 Aql), and with analysis results for SDSSJ0809 (Linnell et al. 2007). We are unaware of a theoretical explanation for the departure of the inner annulus $T_{\text {eff }}$ values from the standard model.

It is possible to start with an assumed $\dot{M}=8 \times 10^{-9} M_{\odot} / \mathrm{yr}^{-1}$ and, by suitably modifying the adopted accretion disk temperature profile, produce an acceptable fit to the observed spectra. But the theoretical connection between the adopted temperature profile and the required energy dissipation budget, to satisfy conservation of energy, becomes more tenuous the larger the departure of the adopted temperature profile from the standard model. The finally adopted mass transfer rate preserves as close a connection to the standard model as possible.

We have verified the unacceptability of the $\dot{M}=8 \times 10^{-9} M_{\odot} / \mathrm{yr}^{-1}$ model by converting 
the temperatures of the inner five boundaries to $29,000 \mathrm{~K}$, the same isothermal value as our adopted model. Since the standard model $T_{\text {eff }}$ varies over a finite width annulus, the Table 8 entries list the $T_{\text {eff }}$ values at the specific radii listed in column 1 . The temperature at the next outer boundary is $25,871 \mathrm{~K}$, so the temperature profile is monotonic. The corresponding synthetic spectrum is a fairly close match to the observed spectral gradient, but the flux still is too high. Changing the normalizing divisor to $1.40 \times 10^{41}$ produces a fit closely similar to Figure 9. However, this normalizing divisor corresponds to a distance to IX Vel of 121 parsecs, slightly more than two standard deviations larger than the Hipparcos distance.

\subsection{An alternative model based on evaporation}

The referee points out a possible alternative model which depends on inner disk evaporation by the hot WD (Lasota 2001). This model would explain why the standard model produces too much blue flux and also why there appears to be no boundary layer in the system.

To evaluate this possibility we started with the standard model and the Table $8 \dot{M}$ and set successively larger inner truncation radii until we found one for which the system synthetic spectrum closely matches the de-reddened $(\mathrm{E}(\mathrm{B}-\mathrm{V})=0.01)$ observed spectra. An inner truncation radius of $2.335 r_{\text {wd }}$ produced a system synthetic spectrum with a fit very similar to Figure 9. The next task is to determine whether the $60,000 \mathrm{~K}$ WD can evaporate the accretion disk to that truncation radius.

Evaporation has been discussed in the context of the quiescent state (Mever \& Mever-Hofmeister 1994; Hameury, Lasota, \& Dubus 1999). This context, with its very low mass transfer rate, is not applicable to IX Vel, whose entire accretion disk is on the hot branch. Other investigations have been in the context of X-ray binaries or black hole systems (Esin. McClintock, \& Naravan 1997; Liu et al. 1999; Manmoto et al. 2000) and Menou et al. (2000). The latter studies provide an evaporation algorithm that permits an estimate of evaporation effects.

The detailed physics of evaporation is poorly understood (Lasota 2001) and it is common to use an ad hoc prescription, such as the expression used by Menou et al. (2000) and Dubus et al. (2001):

$$
\dot{M}_{\text {evap }}(r)=\frac{0.08 \dot{M}_{\mathrm{Edd}}}{\left(r / r_{s}\right)^{1 / 4}+\mathcal{E}\left(r / 800 r_{s}\right)^{2}},
$$

where $\dot{M}_{\mathrm{Edd}}=L_{\mathrm{Edd}} / 0.1 c^{2}=1.39 \times 10^{18} M_{\mathrm{wd}} / M_{\odot} \mathrm{g} \mathrm{s}^{-1}$ is the Eddington rate corresponding to the Eddington luminosity, with a $10 \%$ accretion efficiency. $c$ is the speed of light and $r_{s}=2 G M_{\mathrm{wd}} / c^{2}$ is the Schwarzschild radius. $\mathcal{E}$ is an 'evaporation efficiency' factor, with 
typical values in the range 20 or 30 (Menou et al. 2000) when applied to the case of soft $\mathrm{X}$-ray transients. In the present application the evaporation rate should equal the mass transfer rate from the secondary, evaluated at the truncation radius. The mass transfer rate from the secondary star is $3.2 \times 10^{17} \mathrm{gm} \mathrm{s}^{-1}$. For $\mathcal{E}=20.0, \dot{M}_{\text {evap }}=2.2 \times 10^{13} \mathrm{~g} \mathrm{~s}^{-1}$ at $r / r_{\mathrm{wd}}=2.335$. Based on this model, evaporation is four orders of magnitude too small to produce the required disk truncation.

\subsection{System wind}

IX Vel shows prominent effects of a wind associated with the system (Sion 1985; Mauche 1991; Prinja \& Rosen 1995; Hartley et al. 2002, and L94). Hartley et al. (2002) study whether the IX Vel wind is line-driven. They find that the strong correlation between UV brightness and wind activity predicted by line-driven wind models (Pereyra, Hillier, \& Turnshek 2006) is disobeyed for both IX Vel and V3885 Sgr, and conclude that the most promising alternative to a pure line-driven disk model is a hybrid model including a combination of MHD and line forces. Proga (2003a, b) calculates resonance-line profiles for radiation-driven winds and produces a good fit to the observed C IV $\lambda 1549$ line, with its P Cygni type profile, but requires a mass transfer rate that is six times larger than determined in this study. Other wind lines are much stronger than the C IV $\lambda 1549$ line and do not show P Cygni characteristics.

Long \& Knigge (2002) describe an improved code for spectral synthesis of wind lines in the non-MHD case. See Murray (2002) for a comparison of line-driven winds and MHD driving.

The standard model accounts for $1 / 2$ of the potential energy liberated from the mass transfer stream (Frank. King \& Raine 1992). A boundary layer often is proposed as the mechanism to account for the balance (FKR), but we have seen that IX Vel does not have a hot boundary layer. We assume the system wind carries off the liberated potential energy not accounted for by the accretion disk faces and rim.

\subsection{The bright spot}

Smak (2002), hereafter S2002, discusses the structure of the outer parts of accretion disks with particular reference to tidal heating and radiation from the rim, including effects of a bright spot. S2002 proposes that the luminosity of the rim arises entirely from tidal heating, and consumes all of that source of energy, and that a local bright spot radiates 
away all of the energy released directly by the stream-disk interaction. S2002 references the tidal heating theory in Lasota (2001), and Buat-Ménard, Hameury, \& Lasota (2001). The temperature of the S2002 accretion disk face would be given by the standard model. S2002 points to the study by Ichikawa \& Osaki (1994) which finds that tidal torques are limited to a radially narrow region, producing a sharply-defined accretion disk edge, which S2002 takes to be cylindrical. This model differs from the model we use to define the disk edge (Hubeny \& Plaved 1991). The impact of the mass transfer stream produces strong turbulence and affects a fairly large radial region of the accretion disk (see below). Consequently, we believe the S2002 model is oversimplified.

S2002 calculates a bright spot temperature in the approximate range $18,000 \mathrm{~K}-20,000 \mathrm{~K}$, a range supported by an analysis of UX UMa (Smak 1994) for which there is clear evidence for prompt emission of the energy liberated by the stream-disk collision. This temperature range accords with values determined for other CV systems (Warner 1995, sect. 2.6.5). The $\mathrm{K}$ band photometry rules out a IX Vel bright spot temperature as high as the S2002 model and, instead, limits the bright spot temperature to $\approx 6100 \mathrm{~K}$, only about $500 \mathrm{~K}$ warmer than the rim regions unaffected by the bright spot. (Note that our rim model assigns a rim temperature, $5624 \mathrm{~K}$, somewhat lower than the outermost annulus temperature, 6300K.)

The study by Livio, Soker, \& Dgani (1986) considers the degree of stream penetration into the rim based on their parameter $\alpha$. They show that the stream produces appreciable turbulence in the outer annuli, and that, for small $\alpha$, there can be large penetration, leading to energy release within the outer annuli. Their analysis considers the case in which the stream has a larger diameter than the rim height. We show below that, in IX Vel, the stream diameter is less than the rim height. The ratio of the stream radius, $r_{s}$, to the component separation, D, near the L1 point, is given by the small parameter $\epsilon$ where (Cook \& Warner 1984)

$$
\epsilon \approx 6.1 \times 10^{-3}\left(\frac{T_{\mathrm{eff}}}{10^{3}}\right)^{1 / 2}\left(\frac{M_{2}}{M_{0}}\right)^{-1 / 3}\left(\frac{P_{\mathrm{orb}}}{1 \mathrm{hr}}\right)^{1 / 3}(1+q)^{-1 / 3}, q=M_{2} / M_{1} .
$$

In this equation $T_{\text {eff }}$ applies to the secondary star. In the case of IX Vel, $\epsilon=0.02$ and $r_{s}=2.2 \times 10^{9} \mathrm{~cm}$. From Table 4, the TLUSTY height of the rim is $5.4 \times 10^{9} \mathrm{~cm}$, and the adopted height is about two times this. Thus, the rim height is larger than the stream cross-section and conditions differ from those studied by Livio, Soker, \& Dgani. Różyczka \& Schwarzenberg-Czerny (1987); Różyczka (1988) have developed a hydrodynamical model of the stream-disk interaction. On their model, the stream density at a distance $r$ from the symmetry axis is 


$$
\rho_{s}=\rho_{s c} \exp \left(-r^{2} / r_{s}^{2}\right)
$$

where $\rho_{s c}$ is the stream density on the symmetry axis. Różyczka \& Schwarzenberg-Czerny (1987) set $\rho_{s c}=1.7 \times 10^{-9} \mathrm{gm} \mathrm{cm}^{-3}$ for $\dot{M}=10^{-9} M_{\odot} \mathrm{yr}^{-1}$. For the IX Vel $\dot{M}$, (five times larger) we have $\rho_{s c}=8.5 \times 10^{-9} \mathrm{gm} \mathrm{cm}^{-3}$. The TLUSTY model for the outermost annulus has a central plane density of $1.47 \times 10^{-6} \mathrm{gm} \mathrm{cm}^{-3}$ and a density at the top of $4.25 \times 10^{-14} \mathrm{gm} \mathrm{cm}^{-3}$. The density at $z=2.2 \times 10^{9} \mathrm{~cm}$ (the stream radius) is $5.0 \times 10^{-6} \mathrm{gm} \mathrm{cm}^{-3}$. The top of the annulus is at a height of $6.32 \times r_{s}$. Using equation (5), $\rho_{s}=3.49 \times 10^{-26} \mathrm{gm} \mathrm{cm}^{-3}$ at the rim. Assuming the annulus scale height increases by the same factor as the increased vertical rim dimension, in meeting the geometric requirements to shadow the secondary, we find that the accretion disk rim is everywhere more dense than the stream, consistent with both Różyczka \& Schwarzenberg-Czerny and Livio et al. A basic difference of IX Vel from these two studies is that the rim height is appreciably greater than the stream radius. There do not appear to be any studies of this case.

Pringle (1977) argues that, if the radius of the incident stream is less than the rim semiheight, the impacting stream plows into the rim and dissipates its energy within the accretion disk. An important point made by Pringle (1977) is that, when the stream penetrates deeply, there is a large optical depth to the region of energy dissipation. This argument provides a physical basis to explain why IX Vel has a warm spot rather than a higher temperature spot. It is clear that, for IX Vel, the stream-disk interaction must produce some heating of the outer accretion disk.

The size of the bright spot region is somewhat extended, compared with prominent spots in other CVs. For example, Cook (1985) found an angular width of the spot in OY Car of up to $30^{\circ}$ and an angle of about $5^{\circ}$ between the impact point of the stream and the maximum flux from the spot. Compare with Table 6. Although bright spots in a number of systems associate flickering in those systems (Warner, O'Donoghue \& Allen 1985) with the warm spot (Warner 1995, sect. 2.6.5), there is no basis to connect the flickering observed in IX Vel (Garrison et al. 1984; Eggen \& Niemela 1984; Warner, O’Donoghue \& Allen 1985) with the warm spot.

It is of interest that Kubiak, Pojmanski \& Krzeminski (1999) observe enhanced emission in the Paschen $\mathrm{H}$ lines and the CaII triplet $(8498.02 \AA, 8542.09 \AA, 8662.14 \AA)$ at orbital phase 0.32 , nearly diametrically across the disk from the warm rim region. The enhanced emission parallels other examples of excitation on the opposite side of the disk from the bright spot location (Szkody 1992; Livio 1993; Hillier 2000). Our model would not show significant phase-resolved spectral variation and BINSYN currently does not model emission lines so we have no basis to simulate that observational result. 


\subsection{The annulus extended vertical heights}

It now is generally assumed that the source of viscous dissipation in accretion disks arises

from MRI (Balbus \& Hawley 1991). Hirose, Krolik, \& Stone (2006); Blaes et al. (2006) show that magnetic pressure can be important in vertical hydrostatic support and can lead to vertically extended annuli by about a factor two compared with analytic models. This result is roughly equal to the ratio of our empirically required vertical extent of the outer annulus to the theoretical height based on the TLUSTY models. Since the optical thickness of the annuli is large (see Table 4), the predicted spectrum is quite insensitive to the actual optical thickness of the disk.

\subsection{Caveats}

We note the many uncertainties in the physics of accretion disks and the simplifications required (MHD effects, our adoption of a single viscosity $\alpha$ for the entire accretion disk, our neglect of radial energy transport, our assumption of vertical hydrostatic equilibrium - and consequent neglect of wind effects, etc.). There also are many approximations in our geometrical model (e.g., neglect of the semi-transparent outer layers of the disk when computing the final spectrum).

The intrinsic variability of the source is an important consideration when deciding how worthwhile it is to find a precise fit to our particular set of observations. For example, a discussion within our group raised the question: In view of all the uncertainties listed above, why not simply use the most appropriate Wade \& Hubeny (1998, hereafter WH) accretion disk model, with WD spectrum added, to represent the system? A test with the WH model cc, whose $\dot{M}=3.2 \times 10^{-9} M_{\odot} / \mathrm{yr}^{-1}$, and for an adopted inclination of $60^{\circ}$, showed that a fair fit to the observed spectra could be achieved, with a calculated distance to IX Vel of 89 pc for an added 55,000K WD, only different from the Hipparcos distance by about one standard deviation. And the cc model is a standard model. So, can we maintain the objective reality implied by our final model departure from a standard model temperature profile?

The response in this debate was: The cc model cuts off at $r / r_{\mathrm{wd}, 0}=21.7$ because WH had decided to terminate annulus models at a $T_{\text {eff }}$ of about $10,000 \mathrm{~K}$. The flux error in neglecting the outer accretion disk, as WH note, is of order 9\%. Our calculation, with annuli extending essentially to the tidal cutoff, is not subject to that error. But the more important point is that the tidal cutoff radius is at $r / r_{\mathrm{wd}, 0}=57.1$. Calculation of the full standard model at the model cc mass transfer rate shows that a substantial outer range of the accretion disk has a $T_{\text {eff }}$ below the transition temperature to the low state: the cc model 
is unstable to flux outbursts, where we have taken the transition temperature as 6300K. Recent developments (Lasota 2001; Buat-Ménard, Hameury, \& Lasota 2001) show that the standard model needs correction for tidal and stream impact heating, and these additions affect accretion disk susceptability to flux outbursts. Parenthetically, the WH annuli were calculated with the viscosity represented by a Reynolds number of 5000; models using the now generally adopted Shakura \& Sunyaev (1973) $\alpha=0.1$ can have a very different structure, and would alter the Table 4 entries, although the $T_{\text {eff }}$ values would be the same. Moreover, use of the cc model provides no means to calculate a synthetic light curve, as in Figure 8, and to simulate properties of the accretion disk rim, including its extended vertical height, as our final model has done. Finally, Rutten, van Paradijs, \& Tinbergen (1992) provide observational evidence supporting departures from the standard model. If $\dot{M}$ varies, as the larger (i.e., larger than flickering) IX Vel photometric variability implies, the accretion disk will have a transient response to each change; depending on the transient response time, the accretion disk may well be in a perpetual state of departure from the standard model. It is desirable to have modeling procedures capable of simulating such departures.

The other side in the debate has a valid point: We have not produced an array of models, covering the error ranges of $M_{\mathrm{wd}}, \dot{M}, i$, the Hipparcos distance, etc., in search of an optimum model, together with confidence ellipses for all of the variables involved. It is entirely possible, even likely, that such a search would find other models that fit the observed spectra as well as our final model. The computational effort to do that is completely prohibitive.

What we have done, for the data set we have studied, is to show that a modification of the standard model produces a better fit to the available constraints (observed spectra, $\mathrm{K}$ band light curve, Hipparcos distance) than the standard model, and that our final model is a very good fit to all of the constraints except for a short FUV wavelength region.

\section{Summary}

This analysis of IX Vel leads to the following conclusions:

(1) At the times of the FUSE and STIS spectra studied here, our model derives a mass transfer rate from the secondary star of $\dot{M}=5 \times 10^{-9} M_{\odot} / \mathrm{yr}^{-1}$ and excludes a rate as large as $\dot{M}=8 \times 10^{-9} M_{\odot} / \mathrm{yr}^{-1}$.

(2) It is possible to achieve a close synthetic spectrum fit to the observed FUSE and STIS spectra by adopting an accretion disk temperature profile which agrees with the standard model except for the inner annuli, extending to $r / r_{\mathrm{wd}} \approx 4$. Shortward of that radius the accretion disk is approximately isothermal. 
(3) A light synthesis fit to $\mathrm{K}$ band photometry requires shallow eclipses of the accretion disk rim and secondary star limb. The geometry, including the calculated outer radius of the accretion disk, constrains the orbital inclination to $i=57 \pm 2^{\circ}$ (est.).

(4) A synthetic light curve agreeing with the observed ellipsoidal (not Algol type) variation, requires a vertically extended accretion disk rim to shadow the secondary star, beyond that predicted by gravitational equilibrium. The enhanced vertical extension is consistent with recent MHD predictions for CV accretion disks.

(5) Representation of differing heights of alternate $\mathrm{K}$ band maxima requires a warm rim region downstream of the intersection point of the mass transfer stream with the accretion disk rim. The location and temperature of the warm region are inconsistent with prompt radiation from a bright spot associated with a rim shock, and consistent with deep penetration of the stream.

The authors thank the anonymous referee for carefully reading the manuscript and providing a valuable critique; responding to it has significantly improved the paper presentation. P.S. acknowledges support from FUSE grant NNG04GC97G and HST grant GO-09724. PG is thankful to the Space Telescope Science Institute for its kind hospitality. Support for this work was provided by NASA through grant number HST-AR-10657.01-A to Villanova University (P. Godon) from the Space Telescope Science Institute, which is operated by the Association of Universities for Research in Astronomy, Incorporated, under NASA contact

NAS5-26555. This research was partly based on observations made with the NASA-CNESCSA Far Ultraviolet Spectroscopic Explorer. FUSE is operated for NASA by the Johns Hopkins University under NASA contract NAS5-32958.

\section{REFERENCES}

Allen, C.W. 1973, Astrophysical Quantities 3rd ed. (Clowes \& Sons:Athlone Press)

Balbus, S. A., \& Hawley, J. F. 1991, ApJ, 376, 214

Beuermann, K. \& Thomas, H.-C. 1990, A\&A, 230, 326 (BT)

Blaes, O. M., Davis, S. W., Hirose, S., Krolik, J. H., \& Stone, J.M. 2006, ApJ, 645, 1402

Böhnhardt, H., Dreschel, H., Rahe, J., and Wargau, W. 1982, IAUC 3749

Bruch, A. \& Engel, A. 1994, A\&AS, 1994, 104, 79

Buat-Ménard, V., Hameury, J.-M., \& Lasota, J.-P. 2001, A\&A, 366, 612 
Claret, A. 2001, MNRAS, 327, 989

Cook, M. C. 1985, MNRAS, 215, 211

Cook, M. C. \& Warner, B. 1984, MNRAS, 207, 705

Cox, A. N. (ed.) 2000, Allen's Astrophysical Quantities. 4th edition (New York:Springer)

la Dous, C. 1991, A\&A, 252, 100

Dubus, G., Hameury, J.-M., \& Lasota, J.-P. 2001, A\&A, 373, 251

Eggen, O. J., \& Niemela, V. S. 1984, AJ, 89, 389

Esin, A.A., McClintock, J.E., \& Narayan, R. 1997, ApJ,489, 865

Frank, J., King, A. \& Raine, D. 1992, Accretion Power in Astrophysics (Cambridge: Univ. Press)

Garrison, R. F., Hiltner, W. A., Krzeminski, W., \& Schild, R. E. 1977, ApJS, 35, 111

Garrison, R. F., Hiltner, W. A., Krzeminski, W., \& Schild, R. E. 1982, IAUC 3730

Garrison, R. F., Schild, R. E., Hiltner, W. A. \& Krzeminski, W, 1984, ApJ, 276, L13

Goodman, J. 1993, ApJ, 406, 596

Hameury, J.-M., Lasota, J.-P., \& Dubus, G. 1999, MNRAS, 303, 39

Hartley, L. E., Drew, J. E., Long, K. S., Knigge, C., \& Proga, D. 2002, MNRAS, 332, 127

Haug, K. 1987, Astroph. Sp. Sci., 130, 91

Haug, K. 1988, MNRAS, 235, 1385

Hessman, F. V. 1989, AJ, 98, 675

Hillier, C. 2000, New Astronomy Reviews, 44, 131

Hirose, S., Krolik, J. H., \& Stone, J. M. 2006, ApJ, 640, 901

Holberg, J. B., \& Bergeron, P. 2006, AJ, 132, 1221

Howell, S. B., \& Skidmore, W. 2000, New Astronomy Reviews, 44, 33

Hubeny, I. 1988, Comp. Phys. Comm., 52, 103 
Hubeny, I. 1990, ApJ, 351, 632

Hubeny, I., \& Plavec, M. J. 1991, AJ, 102, 1156

Hubeny, I., Lanz, T., \& Jeffery, C. S. 1994, in Newsletter on Analysis of Astronomical Spectra No. 20, ed. C. S. Jeffery (CCP7;St. Andrews: St. Andrews Univ.), 30

Hubeny, I., \& Lanz, T. 1995, ApJ, 439, 875

Hubeny, I., \& Hubeny, V. 1998, ApJ, 505, 558

Ichikawa, S., \& Osaki, Y. 1994, Pub. Astr. Soc. Japan, 46, 621

Kolb,U., \& Baraffe, I. 2000, New Astronomy Reviews, 44, 99

Kubiak, M., Pojmanski, G., \& Krzeminski, W. 1999, Acta Astron., 49, 73

Lasota, J.-P. 2001, New Astronomy Reviews, 45, 449

Linnell, A. P., \& Hubeny, I. 1996, ApJ, 471, 958

Linnell, A. P., Hoard, D. W., Szkody, P., Long, K. S. Hubeny, I., Gänsicke, B., \& Sion, E. M. 2007, ApJ, 654, 1036

Liu, B.F., Yuan, W., Meyer, F., Meyer-Hofmeister, E., Xie, G.Z. 1999, ApJ, 527, 17

Livio, M. 1993, in Accretion Disks in Compact Stellar Systems, ed. J.C. Wheeler (Singapore: World Scientific).

Livio, M., Soker, N., \& Dgani, R. 1986, ApJ, 305, 267

Long, K. S., Wade, R. A., Blair, W. P., Davidsen, A. F., and Hubeny, I. 1994, ApJ, 426, 704 (L94)

Long, K. S., \& Knigge, C. 2002, in The Physics of Cataclysmic Variables and Related Objects, ASP Conference Series, vol. 261, p. 327 eds. B. T. Gänsicke, K. Beuermann, and K. Reinsch (San Francisco: The Astronomical Society of the Pacific)

Lubow, S. H., \& Shu, F. H. 1975, ApJ, 198, 383

Manmoto, T., Kato, S., Nakamura, K.E., \& Narayan, R. 2000, ApJ, 529, 127

Mauche, C. W. 1991, ApJ, 373, 624

Mauche, C. W., Raymond, J. C., and Córdova, F. A. 1988, ApJ, 335, 829 
Menou, K., Hameury, J.-M., Lasota, J.-P., \& Narayan, R. 2000, MNRAS, 314, 498

Meyer, F. \& Meyer-Hofmeister, E. 1994, A\&A, 1994, 288, 175

Murray, N. 2002, in The Physics of Cataclysmic Variables and Related Objects, ASP Conference Series, vol. 261, p. 308 eds. B. T. Gänsicke, K. Beuermann, and K. Reinsch (San Francisco: The Astronomical Society of the Pacific)

Osaki, Y. 1996, PASP, 108, 39

Paczynski, B. 1977, ApJ, 216, 822

Panei, J. A., Althaus, L. G., \& Benvenuto, O. G. 2000, A\&A, 353, 970

Papaloizou, J., \& Pringle, J. E. 1977, MNRAS, 181, 441

Pereyra, N. A., Hillier, D. J., \& Turnshek, D. A. 2006, ApJ, 636, 411

Perryman,M. A. C. et.al. 1997, A\&A, 323L, 49P

Pojmaǹski, G., \& Udalski, A. 1997, Acta Astron., 47, 451

Polidan, R. S., Mauche, C. W., \& Wade, R. A. 1990, ApJ, 356, 211

Pringle, J. E. 1977, MNRAS, 178, 195

Prinja, R. K. \& Rosen, S. R. 1995, MNRAS, 273, 461

Proga, D. 2003a, ApJ, 585, 406

Proga, D. 2003b, ApJ, 592, L9

Różyczka, M. \& Schwarzenberg-Czerny, A. 1987, Acta Astron., 37, 141

Różyczka, M. 1988, Acta Astron., 38, 175

Rutten, R. G. M., van Paradijs, J., \& Tinbergen, J. 1992, A\&A, 260, 213

Schwarzenberg-Czerny, A. \& Różyczka, M. 1988, Acta Astron., 38, 189

Shakura, N. I., \& Sunyaev, R. A. 1973, A\&A, 24, 337

Shaviv, G., \& Wehrse, R. 1991, A\&A, 251, 117

Sion, E. M. 1985, ApJ, 292, 601

Smak, J. 1982, Acta Astron., 32, 199 
Smak, J. 1994, Acta Astron., 44, 59

Smak, J. 2002, Acta Astron., 52, 263

Szkody, P. 1992, in Viña Del Mar Workshop on Cataclysmic Variable Stars, ASP Conference Series vol. 29 (San Francisco: The Astronomical Society of the Pacific), p.42

van Teeseling, A., Drake, J. J., Drew, J. E., Hoare, M. G., \& Verbunt, F. 1995, A\&A, 300, 808

Verbunt, F. 1987, A\&AS, 71, 339

Wade, R. A., \& Rucinski, S. M. 1985, A\&AS, 60, 471

Wade, R.A. \& Hubeny, I. 1998, ApJ, 509, 350

Wargau, W., Dreschel, H., Rahe, J., \& Bruch, A. 1983, MNRAS, 204, 35p

Wargau, W., Bruch, A., Dreschel, H., Rahe, J., \& Schoembs, R. 1984, Ap\&SS, 94, 145

Warner, B. 1995, Cataclysmic Variable Stars (Cambridge: University Press)

Warner, B., O’Donoghue, D., \& Allen, S. 1985, MNRAS, 212, 9p

Whitehurst, R. 1988, MNRAS, 233, 529

Whitehurst, R., \& King, A. 1991, MNRAS, 249, 25 
Table 1: FUV Observations of IX Vel: HST \& FUSE Spectra

\begin{tabular}{clcllll}
\hline $\begin{array}{c}\text { Date } \\
(\mathrm{dd} / \mathrm{mm} / \text { yyyy })\end{array}$ & $\begin{array}{l}\text { Telescope } \\
\text { /nstrument }\end{array}$ & $\begin{array}{c}\text { Exp time } \\
(\mathrm{sec})\end{array}$ & Dataset & $\begin{array}{l}\text { Filter/Grating } \\
\text { /Aperture }\end{array}$ & $\begin{array}{l}\text { Operation } \\
\text { Mode }\end{array}$ & $\begin{array}{l}\text { Calibration } \\
\text { Software }\end{array}$ \\
\hline 15-04-2000 & FUSE & 7336 & Q1120101 & LWRS & HIST & CalFUSE 3.0.7 \\
$19-07-2000$ & STIS & 1750 & o5bi03010 & E140M/0.1x0.03 & TIME-TAG & CALSTIS 2.22 \\
\hline
\end{tabular}


Table 2: FUSE Lines

\begin{tabular}{|c|c|c|}
\hline $\begin{array}{l}\text { Line } \\
\text { Identification }\end{array}$ & $\begin{array}{l}\text { Wavelength } \\
(\AA)\end{array}$ & Origin \\
\hline H I & see text & $\mathrm{s}, \mathrm{c}, \mathrm{ism}$ \\
\hline O I & see text & a,c,ism \\
\hline N IV & $921.5-924.9$ & $\mathrm{~S}$ \\
\hline S VI & 933.5 & $\mathrm{~S}$ \\
\hline S VI & 944.5 & $\mathrm{~S}$ \\
\hline \multirow[t]{2}{*}{ N I } & 952.4-954.1 & a,c,ism \\
\hline & $964.0-965.04$ & $\mathrm{c}, \mathrm{ism}$ \\
\hline C III & 977.0 & $\mathrm{~s}$ \\
\hline N III & 991.6 & $\mathrm{~s}$ \\
\hline \multirow[t]{2}{*}{ O vi } & 1031.9 & $\mathrm{~S}$ \\
\hline & 1037.6 & $\mathrm{~S}$ \\
\hline $\mathrm{C}$ II & 1036.3 & ism \\
\hline \multirow[t]{2}{*}{ Ar I } & 1048.2 & ism \\
\hline & 1066.7 & ism \\
\hline S IV & 1062.6 & $\mathrm{~S}$ \\
\hline \multirow[t]{2}{*}{ S IV } & 1073.0 & $\mathrm{~s}$ \\
\hline & 1073.5 & $\mathrm{~s}$ \\
\hline $\mathrm{N}$ II & 1084.0 & a,c,ism \\
\hline S IV, VI & 1117.8 & $\mathrm{~s}$ \\
\hline \multirow[t]{2}{*}{$\mathrm{P}_{\mathrm{V}}$} & 1118.0 & $\mathrm{~S}$ \\
\hline & 1128.0 & $\mathrm{~S}$ \\
\hline \multirow[t]{2}{*}{ Si IV } & 1122.5 & $\mathrm{~S}$ \\
\hline & 1128.3 & $\mathrm{~S}$ \\
\hline $\mathrm{N} \mathrm{I}$ & $1134.2-1135.0$ & a,c,ism \\
\hline Fe II & 1144.9 & $\mathrm{c}, \mathrm{ism}$ \\
\hline C III & 1174.9-1176.4 & $\mathrm{s}$ \\
\hline
\end{tabular}

$\mathrm{a}=$ possibly with terrestrial contamination; $\mathrm{c}=$ circumbinary material; ism=ISM; $\mathrm{s}=$ source 
Table 3: STIS Lines

\begin{tabular}{lll}
\hline Line & $\begin{array}{l}\text { Wavelength } \\
\text { Identification }\end{array}$ & Origin \\
\hline C III & $1174.9-1176.4$ & $\mathrm{~s}$ \\
Si II & 1190.4 & $\mathrm{c}$, ism \\
& 1193.3 & $\mathrm{c}$, ism \\
& 1194.5 & $\mathrm{c}$, ism \\
N I & $1199.5-1200.7$ & $\mathrm{c}$, ism \\
Si III & 1206.5 & $\mathrm{c}$ \\
H I & 1215.7 & $\mathrm{~s}$ \\
N V & 1238.8 & $\mathrm{~s}$ \\
& 1242.8 & $\mathrm{~s}$ \\
S II & 1250.6 & $\mathrm{c}$, ism \\
& 1253.8 & $\mathrm{c}$, ism \\
& 1259.5 & $\mathrm{c}$, ism \\
0 I & 1260.4 & $\mathrm{c}$, ism \\
& 1302.2 & $\mathrm{c}$, ism \\
Hl II & 1304.9 & $\mathrm{c}$, ism \\
Si II & 1306.0 & $\mathrm{c}$, ism \\
C II & 1334.5 & $\mathrm{c}$, ism \\
& 1335.7 & $\mathrm{c}$, ism \\
& 1393.8 & $\mathrm{~s}$ \\
& 1402.8 & $\mathrm{~s}$ \\
& 1426.4 & $\mathrm{c}$, ism \\
& 1526.7 & $\mathrm{c}$, ism \\
& 1533.4 & $\mathrm{c}$, ism \\
& 1548.2 & $\mathrm{~s}$ \\
& 1550.8 & $\mathrm{~s}$ \\
& 1640.5 & $\mathrm{~s}$ \\
& & $\mathrm{c}$, ism \\
\hline & & \\
& &
\end{tabular}

$\mathrm{c}=$ circumbinary material; ism $=\mathrm{ISM} ; \mathrm{s}=$ source 
Table 4. Properties of accretion disk with mass transfer rate $\dot{M}=5.0 \times 10^{-9} M_{\odot} \mathrm{yr}^{-1}$ and WD mass of $0.80 M_{\odot}$.

\begin{tabular}{rrcrcccc}
\hline \hline$r / r_{\text {wd }, 0}$ & \multicolumn{1}{c}{$T_{\text {eff }}$} & $m_{0}$ & \multicolumn{1}{c}{$T_{0}$} & $\log g$ & $z_{0}$ & $N e$ & $\tau_{\text {Ross }}$ \\
\hline 1.36 & 59317 & $1.213 \mathrm{E} 4$ & 39973 & 6.97 & $7.81 \mathrm{E} 7$ & $9.68 \mathrm{E} 11$ & $1.99 \mathrm{E} 4$ \\
2.00 & 53184 & $1.481 \mathrm{E} 4$ & 37066 & 6.71 & $1.35 \mathrm{E} 8$ & $5.68 \mathrm{E} 11$ & $2.37 \mathrm{E} 4$ \\
3.00 & 43006 & $1.447 \mathrm{E} 4$ & 31573 & 6.41 & $2.29 \mathrm{E} 8$ & $3.35 \mathrm{E} 11$ & $2.80 \mathrm{E} 4$ \\
4.00 & 36147 & $1.347 \mathrm{E} 4$ & 24987 & 6.19 & $3.25 \mathrm{E} 8$ & $2.47 \mathrm{E} 11$ & $3.23 \mathrm{E} 4$ \\
5.00 & 31354 & $1.251 \mathrm{E} 4$ & 19127 & 6.02 & $4.24 \mathrm{E} 8$ & $2.13 \mathrm{E} 11$ & $3.67 \mathrm{E} 4$ \\
6.00 & 27816 & $1.169 \mathrm{E} 4$ & 15966 & 5.87 & $5.28 \mathrm{E} 8$ & $1.84 \mathrm{E} 11$ & $4.15 \mathrm{E} 4$ \\
8.00 & 22919 & $1.037 \mathrm{E} 4$ & 13021 & 5.65 & $7.47 \mathrm{E} 8$ & $1.34 \mathrm{E} 11$ & $5.17 \mathrm{E} 4$ \\
10.00 & 19661 & $9.380 \mathrm{E} 3$ & 11433 & 5.48 & $9.76 \mathrm{E} 8$ & $1.01 \mathrm{E} 11$ & $6.28 \mathrm{E} 4$ \\
12.00 & 17318 & $8.610 \mathrm{E} 3$ & 10386 & 5.34 & $1.21 \mathrm{E} 9$ & $7.80 \mathrm{E} 10$ & $7.42 \mathrm{E} 4$ \\
14.00 & 15542 & $7.990 \mathrm{E} 3$ & 9599 & 5.22 & $1.46 \mathrm{E} 9$ & $6.34 \mathrm{E} 10$ & $8.53 \mathrm{E} 4$ \\
16.00 & 14143 & $7.479 \mathrm{E} 3$ & 8970 & 5.11 & $1.70 \mathrm{E} 9$ & $5.31 \mathrm{E} 10$ & $9.50 \mathrm{E} 4$ \\
18.00 & 13009 & $7.048 \mathrm{E} 3$ & 8448 & 5.02 & $1.95 \mathrm{E} 9$ & $4.53 \mathrm{E} 10$ & $1.02 \mathrm{E} 5$ \\
20.00 & 12068 & $6.679 \mathrm{E} 3$ & 8000 & 4.93 & $2.19 \mathrm{E} 9$ & $3.91 \mathrm{E} 10$ & $1.06 \mathrm{E} 5$ \\
24.00 & 10591 & $6.076 \mathrm{E} 3$ & 8594 & 4.77 & $2.64 \mathrm{E} 9$ & $2.60 \mathrm{E} 10$ & $1.06 \mathrm{E} 5$ \\
30.00 & 9019 & $5.401 \mathrm{E} 3$ & 6478 & 4.59 & $3.37 \mathrm{E} 9$ & $1.74 \mathrm{E} 10$ & $9.85 \mathrm{E} 4$ \\
35.00 & 8067 & $4.973 \mathrm{E} 3$ & 6546 & 4.46 & $3.82 \mathrm{E} 9$ & $1.37 \mathrm{E} 10$ & $9.37 \mathrm{E} 4$ \\
40.00 & 7322 & $4.626 \mathrm{E} 3$ & 5945 & 4.42 & $4.35 \mathrm{E} 9$ & $6.55 \mathrm{E} 09$ & $9.14 \mathrm{E} 4$ \\
45.00 & 6721 & $4.338 \mathrm{E} 3$ & 5470 & 4.39 & $4.90 \mathrm{E} 9$ & $2.26 \mathrm{E} 09$ & $9.40 \mathrm{E} 4$ \\
50.00 & 6224 & $4.094 \mathrm{E} 3$ & 5067 & 4.13 & $5.41 \mathrm{E} 9$ & $6.81 \mathrm{E} 08$ & $1.05 \mathrm{E} 5$ \\
\hline
\end{tabular}

Note. - Each line in the table represents a separate annulus. A Shakura \& Sunyaev (1973) viscosity parameter $\alpha=0.1$ was used in calculating all annuli. The WD radius, $r_{\mathrm{wd}, 0}$, is the radius of a zero temperature Hamada-Salpeter carbon model. 
Table 5. IX Vel Model System Parameters, Beuermann \& Thomas (1990)

\begin{tabular}{llll}
\hline \hline parameter & \multicolumn{1}{c}{ value } & parameter & \multicolumn{1}{c}{ value } \\
\hline$M_{\mathrm{wd}}$ & $0.80 \pm 0.15 M_{\odot}$ & $i$ & $60 \pm 5^{\circ}$ \\
$M_{2}$ & $0.52 \pm 0.10 M_{\odot}$ & $K_{\mathrm{wd}}$ & $138 \mathrm{~km} \mathrm{~s}^{-1}$ \\
$\dot{M}$ & $7.9 \pm 1.0 \times 10^{-9} M_{\odot} \mathrm{yr}^{-1}$ & $K_{2}$ & $212 \mathrm{~km} \mathrm{~s}^{-1}$ \\
$\mathrm{P}$ & 0.1939292 days & $T_{\mathrm{eff}, 2}$ & $3500 \mathrm{~K}$ \\
$d$ & $95 \pm 12 \mathrm{pc}$ & & \\
\hline
\end{tabular}


Table 6. Properties of accretion disk rim model, $\mathrm{K}$ band light curve

\begin{tabular}{lrlrlr}
\hline \hline parameter & value & parameter & value & parameter & value \\
\hline$T_{\text {eff,rim }}$ & $5624 \mathrm{~K}$ & HSAZ & 270 & HWDW & 30 \\
HSTEMP & $6.1 \mathrm{~K}$ & HSDWD & 10 & TDWND & $5.7 \mathrm{~K}$ \\
HSUP & 10 & TUPD & $5.7 \mathrm{~K}$ & & \\
\hline
\end{tabular}

Note. - HSAZ is the position angle (degrees) of the center of the bright spot region. HWDW is the angular width of the bright spot, in degrees. HSTEMP is the temperature of the bright spot. HSDWD is the angular width (degrees) of the downwind region from the end of the bright spot to the merge point with the rim proper. TDWND is the temperature of the middle of region HSDWD. HSUP is the angular width (degrees) of the upwind region comparable to HSDWD. TUPD is the temperature of the middle of region HSUP. The value of $T_{\text {eff, rim }}$ corresponds, according to equations $(1),(2)$ to an outer annulus $T_{\text {eff }}$ of $6300 \mathrm{~K}$. 
Table 7. Comparison of temperature profiles, adopted and standard model, for accretion disk with mass transfer rate of $\dot{M}=5.0 \times 10^{-9} M_{\odot} \mathrm{yr}^{-1}$ and WD mass of $0.80 M_{\odot}$.

\begin{tabular}{rrrrrr}
\hline \hline$r / r_{\text {wd, } 0}$ & $T_{\text {eff }}($ adopt, K) & $T_{\text {eff }}($ std, K $)$ & $r / r_{\text {wd, }, 0}$ & $T_{\text {eff }}($ adopt, $\mathrm{K})$ & $T_{\text {eff }}($ std, K $)$ \\
\hline 1.00 & 30000 & 54961 & 29.23 & 9206 & 9206 \\
1.18 & 30000 & 55252 & 31.08 & 8805 & 8805 \\
1.36 & 30000 & 59557 & 32.94 & 8443 & 8443 \\
3.22 & 30000 & 41366 & 34.80 & 8114 & 8114 \\
5.08 & 30000 & 31111 & 36.66 & 7814 & 7814 \\
6.93 & 25301 & 25301 & 38.51 & 7639 & 7639 \\
8.79 & 21527 & 21527 & 40.37 & 7285 & 7285 \\
10.65 & 18856 & 18856 & 42.23 & 7051 & 7051 \\
12.51 & 16854 & 16854 & 44.09 & 6833 & 6833 \\
14.36 & 15291 & 15291 & 45.94 & 6631 & 6631 \\
16.22 & 14030 & 14030 & 47.80 & 6442 & 6442 \\
18.08 & 12991 & 12991 & 49.66 & 6300 & 6266 \\
19.94 & 12116 & 12116 & 51.52 & 6300 & 6100 \\
21.80 & 11368 & 11368 & 53.38 & 6300 & 5944 \\
23.65 & 10720 & 10720 & 55.23 & 6300 & 5797 \\
25.51 & 10154 & 10154 & 57.09 & 6300 & 5659 \\
27.37 & 9653 & 9653 & & & \\
\hline
\end{tabular}

Note. - The WD radius, $r_{\mathrm{wd}, 0}$, is the radius of a zero temperature HamadaSalpeter carbon model. 
Table 8. Comparison of temperature profiles, adopted and standard model, for accretion disk with mass transfer rate of $\dot{M}=5.0 \times 10^{-9} M_{\odot} \mathrm{yr}^{-1}$ and WD mass of $0.80 M_{\odot}$.

\begin{tabular}{rrrrrr}
\hline \hline$r / r_{\text {wd }}$ & $T_{\text {eff }}($ adopt, K) & $T_{\text {eff }}($ std, $\mathrm{K})$ & $r / r_{\text {wd }}$ & $T_{\text {eff }}($ adopt, $\mathrm{K})$ & $T_{\text {eff }}($ std, K) \\
\hline 1.00 & 29000 & 41632 & 19.36 & 9015 & 9015 \\
1.18 & 29000 & 41632 & 20.56 & 8636 & 8636 \\
1.36 & 29000 & 43303 & 21.76 & 8293 & 8293 \\
2.56 & 29000 & 34310 & 22.96 & 7980 & 7980 \\
3.76 & 27415 & 27415 & 24.16 & 7694 & 7694 \\
4.96 & 23003 & 23003 & 25.36 & 7430 & 7430 \\
6.16 & 19950 & 19950 & 26.56 & 7187 & 7187 \\
7.36 & 17703 & 17703 & 27.77 & 6962 & 6962 \\
8.56 & 15972 & 15972 & 28.97 & 6753 & 6753 \\
9.76 & 14594 & 14594 & 30.17 & 6558 & 6558 \\
10.96 & 13466 & 13466 & 31.37 & 6376 & 6376 \\
12.16 & 12524 & 12524 & 32.57 & 6300 & 6204 \\
13.36 & 11724 & 11724 & 33.77 & 6300 & 6044 \\
14.56 & 11035 & 11035 & 34.97 & 6300 & 5893 \\
15.76 & 10434 & 10434 & 36.17 & 6300 & 5751 \\
16.96 & 9906 & 9906 & 37.37 & 6300 & 5616 \\
18.16 & 9436 & 9436 & & & \\
\hline
\end{tabular}

Note. $-r_{\mathrm{wd}}$ in the first column heading represents the radius of a $60,000 \mathrm{~K}$ carbon WD. 
Table 9. IX Vel Model System Parameters

\begin{tabular}{llll}
\hline \hline parameter & \multicolumn{1}{c}{ value } & parameter & \multicolumn{1}{c}{ value } \\
\hline$M_{\mathrm{wd}}$ & $0.80 \pm 0.2 M_{\odot}$ & $T_{\text {eff,s }}($ pole $)$ & $3500 \pm 1000 \mathrm{~K}$ \\
$M_{\mathrm{sec}}$ & $0.52 \pm 0.10 M_{\odot}$ & $T_{\text {eff,s }}($ point $)$ & $3119 \mathrm{~K}$ \\
$\dot{M}$ & $5.0 \pm 1.0 \times 10^{-9} M_{\odot} \mathrm{yr}^{-1}$ & $T_{\text {eff }, \mathrm{s}}($ side $)$ & $3451 \mathrm{~K}$ \\
$\mathrm{P}$ & 0.1939292 days & $T_{\text {eff, }}($ back $)$ & $3345 \mathrm{~K}$ \\
$D$ & $1.54588 R_{\odot}$ & $r_{s}($ pole $)$ & $0.496 R_{\odot}$ \\
$\Omega_{\mathrm{wd}}$ & 103.8 & $r_{s}($ point $)$ & $0.705 R_{\odot}$ \\
$\Omega_{s}$ & 3.15422 & $r_{s}($ side $)$ & $0.3518 R_{\odot}$ \\
$i$ & $57 \pm 2^{\circ}$ & $r_{s}$ back $)$ & $0.568 R_{\odot}$ \\
$T_{\text {eff,wd }}$ & $60,000 \pm 10000 \mathrm{~K}$ & $\log g_{s}($ pole $)$ & 4.78 \\
$r_{\mathrm{wd}}$ & $0.0150 R_{\odot}$ & $\log g_{s}($ point $)$ & -4.63 \\
$\log g_{\mathrm{wd}}$ & 7.99 & $\log g_{s}($ side $)$ & 4.70 \\
$A_{\mathrm{wd}}$ & 1.0 & $\log g_{s}($ back $)$ & 4.54 \\
$A_{s}$ & 0.6 & $r_{a}$ & $0.56 R_{\odot}$ \\
$\beta_{\mathrm{wd}}$ & 0.25 & $r_{b}$ & $0.00985 R_{\odot}$ \\
$\beta_{s}$ & 0.08 & $H$ & $0.20 R_{\odot}$ \\
\hline
\end{tabular}

Note. - wd refers to the WD; $s$ refers to the secondary star. $D$ is the component separation of centers, $\Omega$ is a Roche potential. Temperatures are polar values, $A$ values are bolometric albedos, and $\beta$ values are gravity-darkening exponents. $r_{a}$ specifies the outer radius of the accretion disk, set at the tidal cut-off radius, and $r_{b}$ is the accretion disk inner radius, as determined in the final system model, while $H$ is the semi-height of the accretion disk rim. 

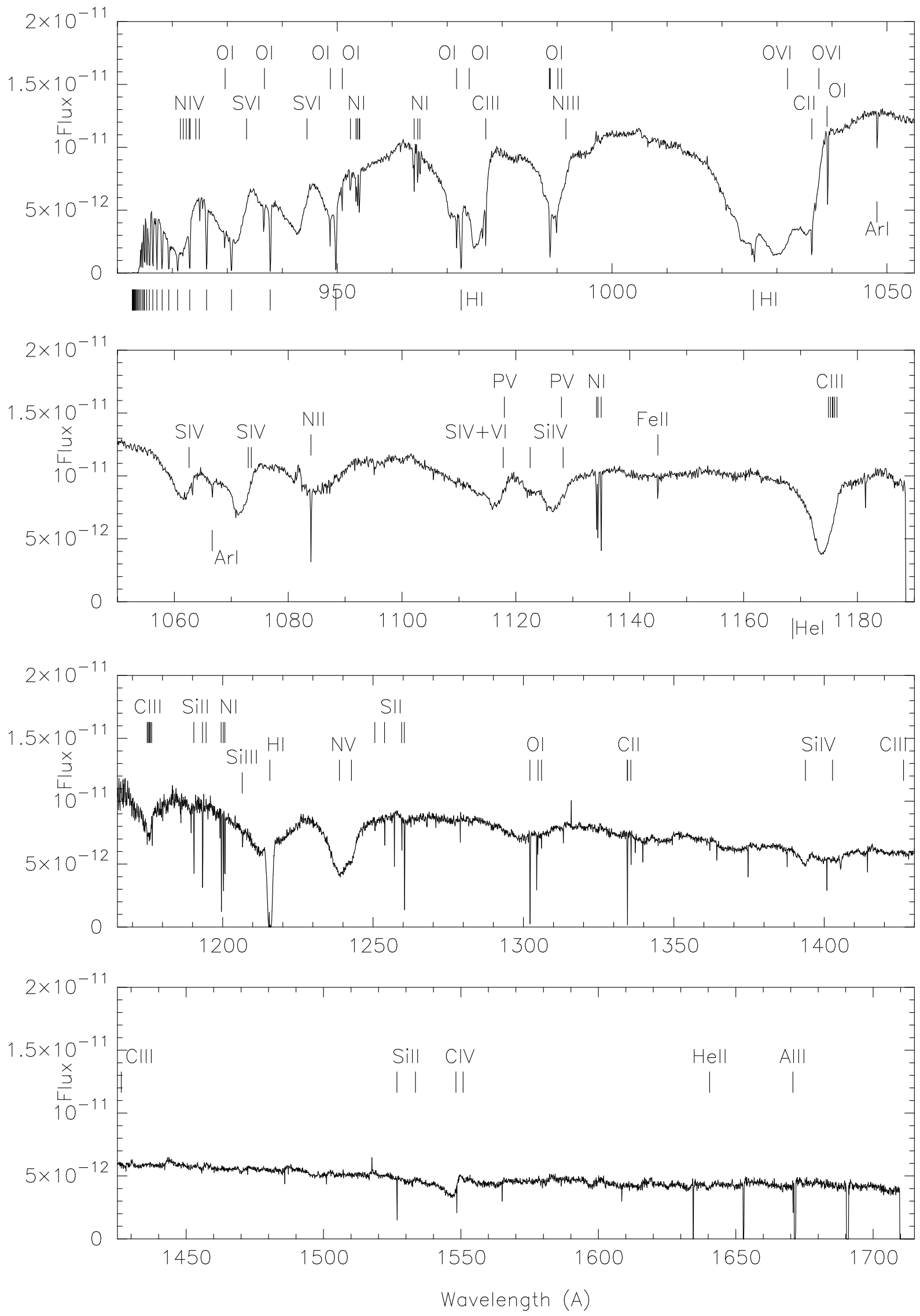

Fig. 1. - FUSE plus STIS combined spectra with line identifications. 


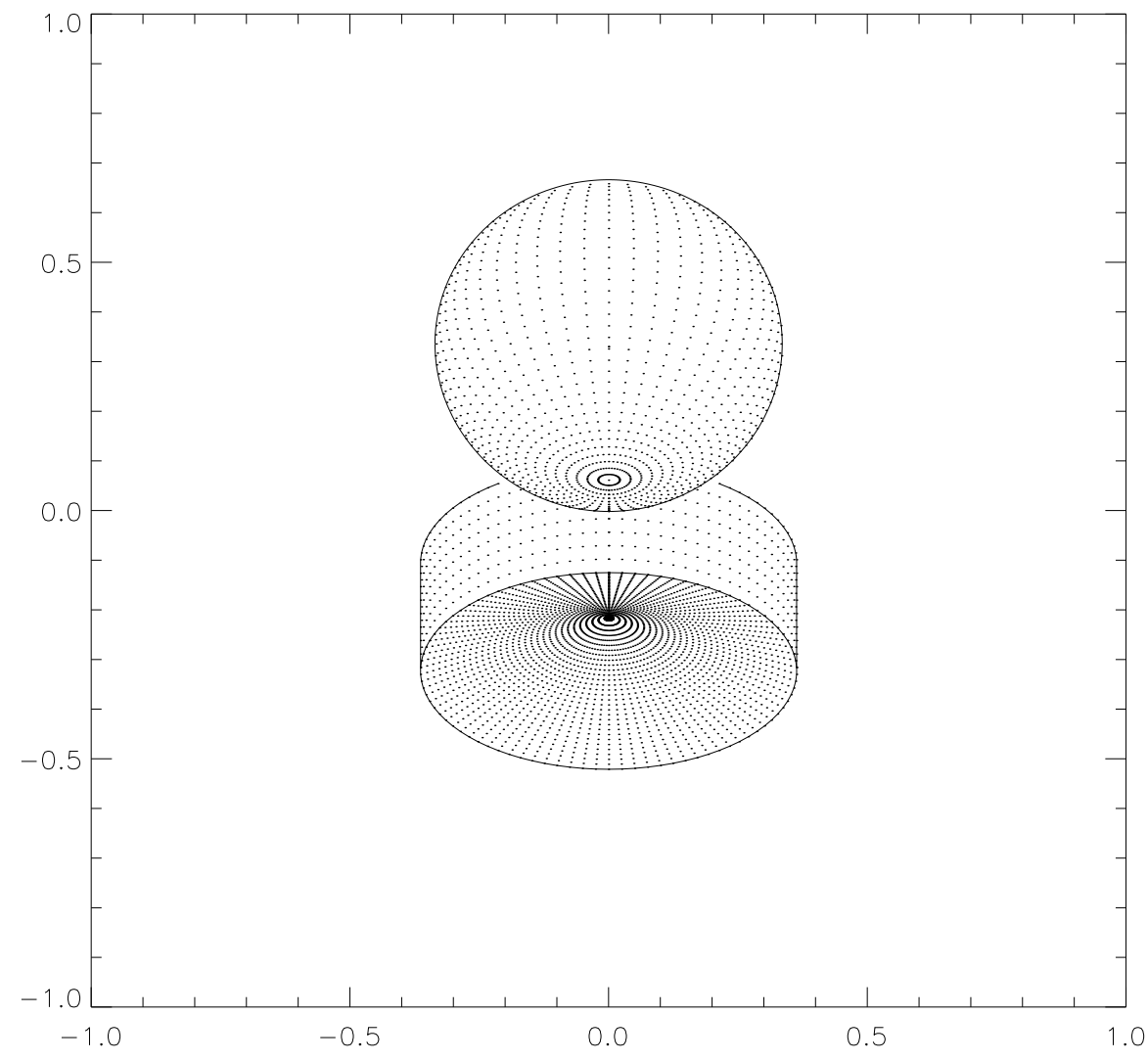

Fig. 2.- IX Vel model projected on the plane of the sky. The orbital inclination is $57^{\circ}$ and the orbital phase is 0.0 . 


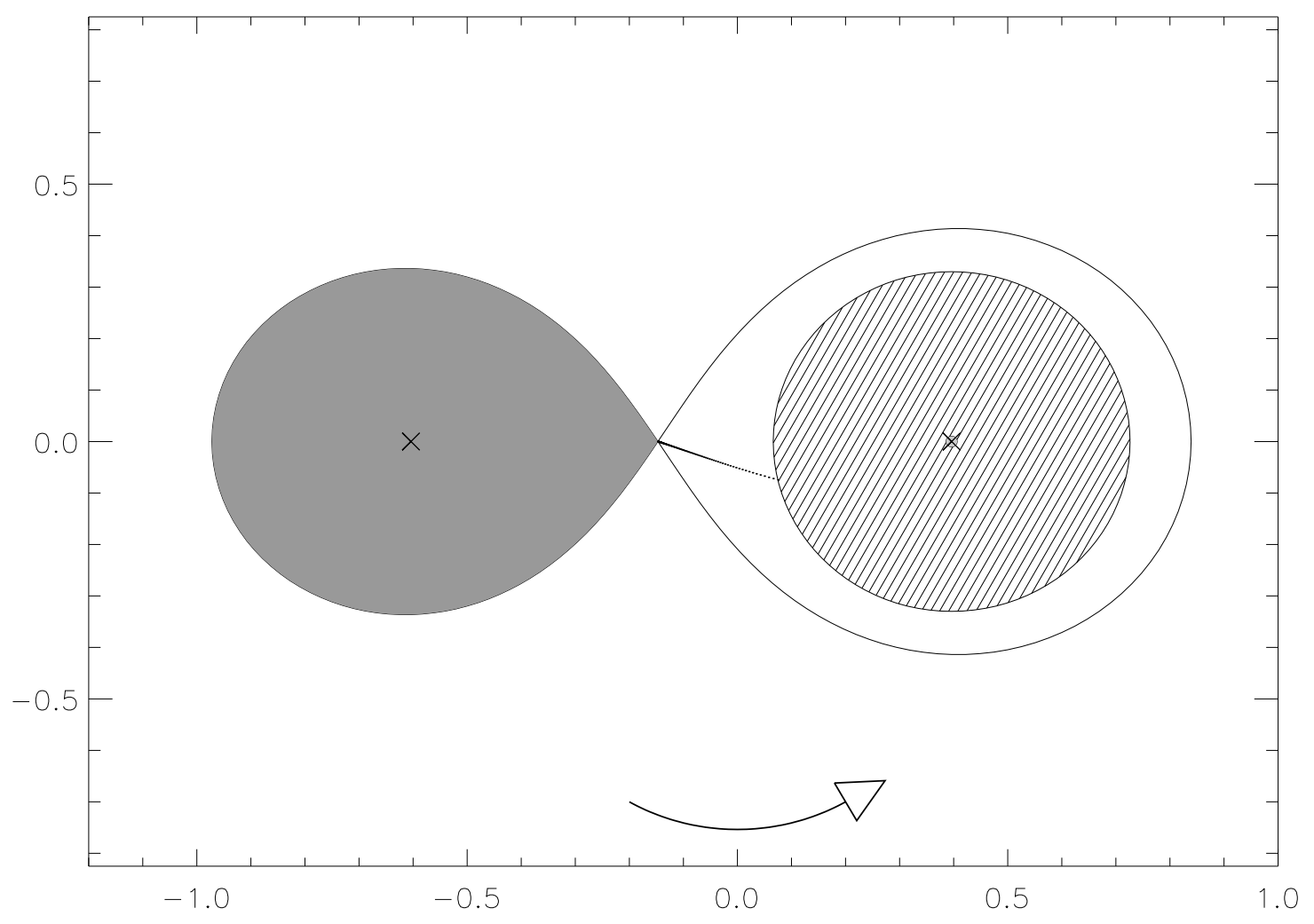

Fig. 3. - Plan view of IX Vel system. The mass stream trajectory follows the theory of Lubow \& Shu (1975). See the text for a discussion. 


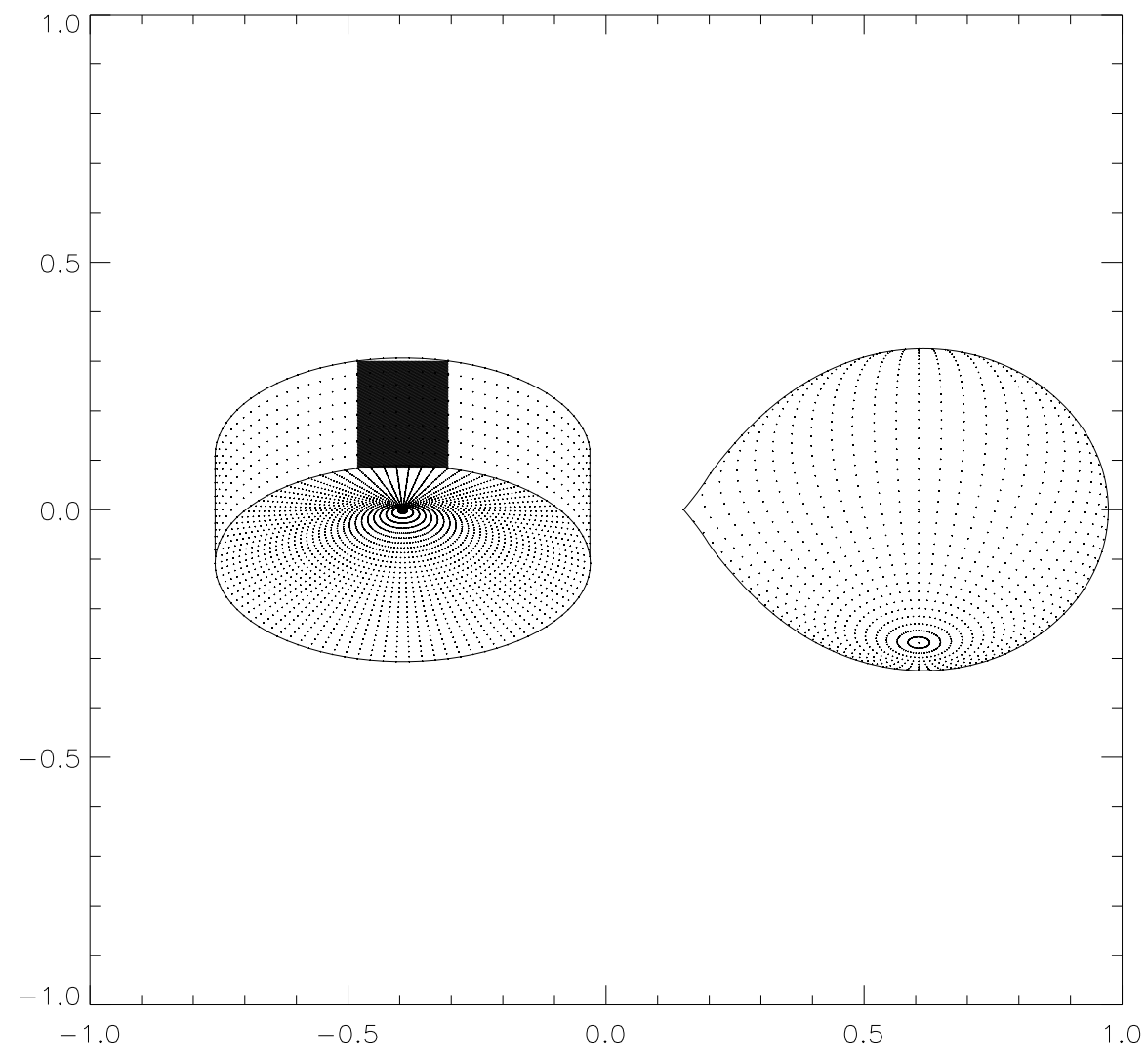

Fig. 4.- IX Vel model at orbital phase 0.75. The dark rim region marks the bright spot, not shown on Figure 2. The bright region is downwind from the point of impact of the mass transfer stream. 


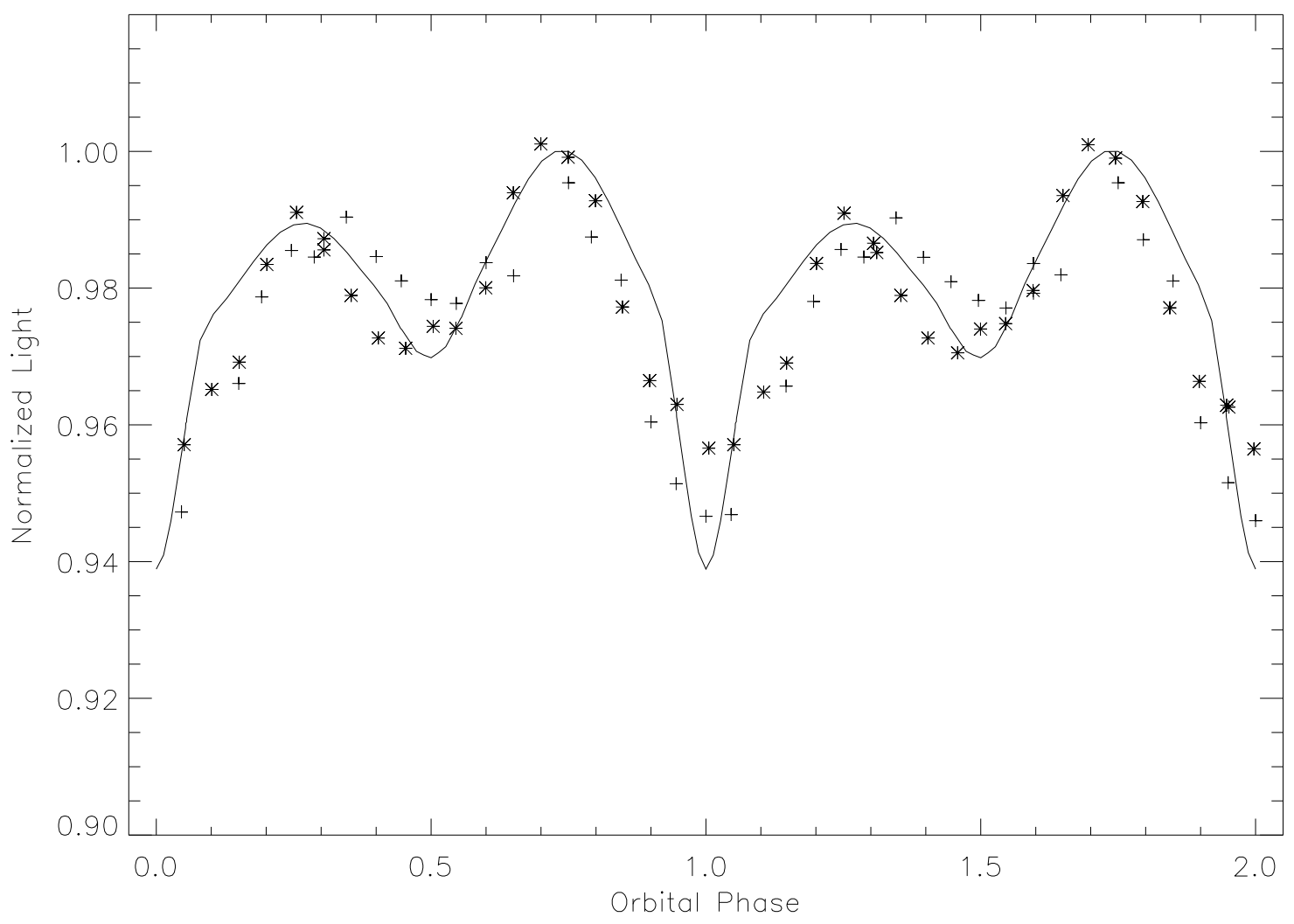

Fig. 5.- Synthetic light curve and observed (binned) K band observations for an orbital inclination of $57^{\circ}$. The crosses mark observations of 1984 January 16-23; the asterisks mark observations of 1987 January 12-16. See the text for details. 


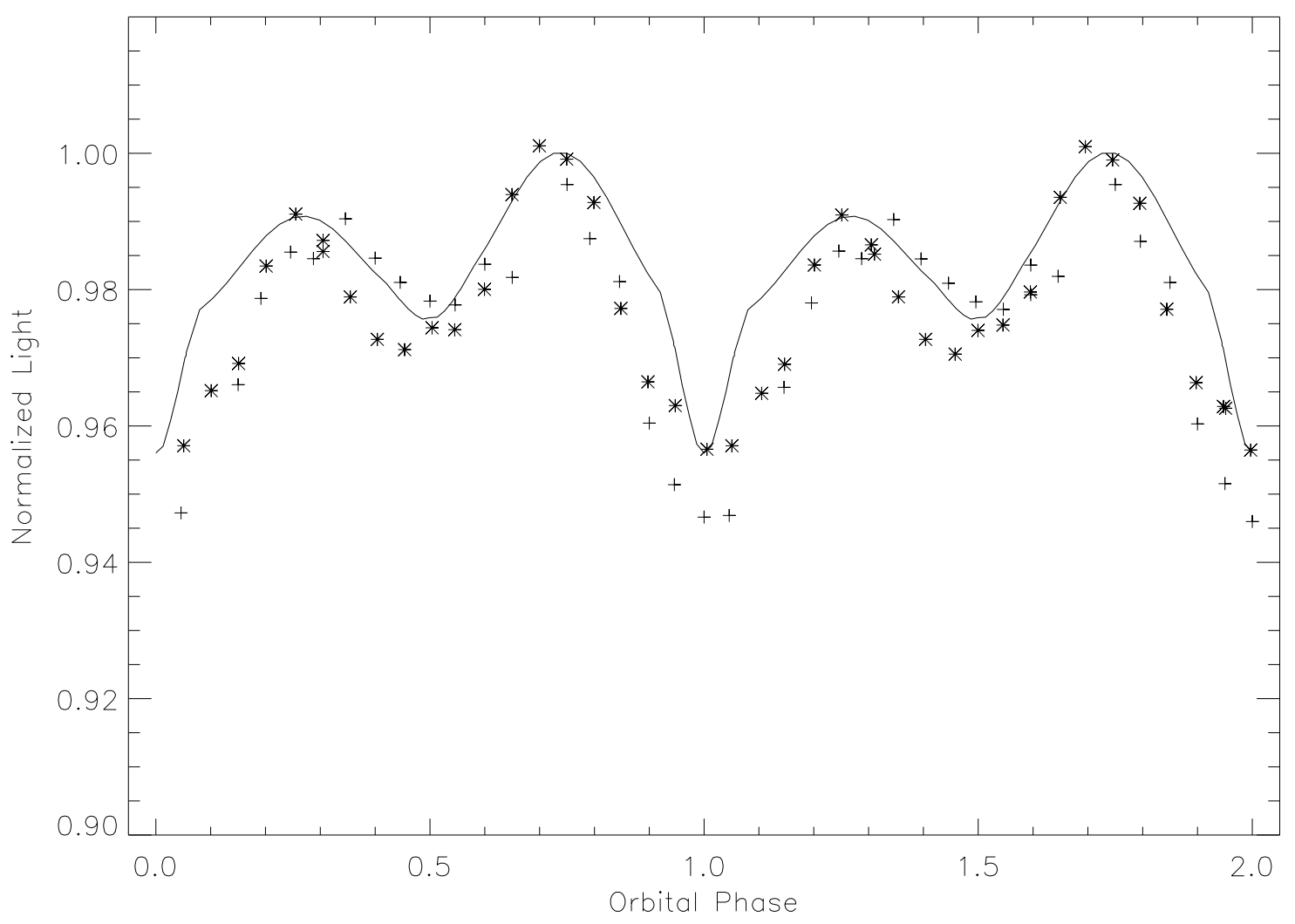

Fig. 6.- As in Figure 5 but for an orbital inclination of $54^{\circ}$. The light curve amplitude is too small. 


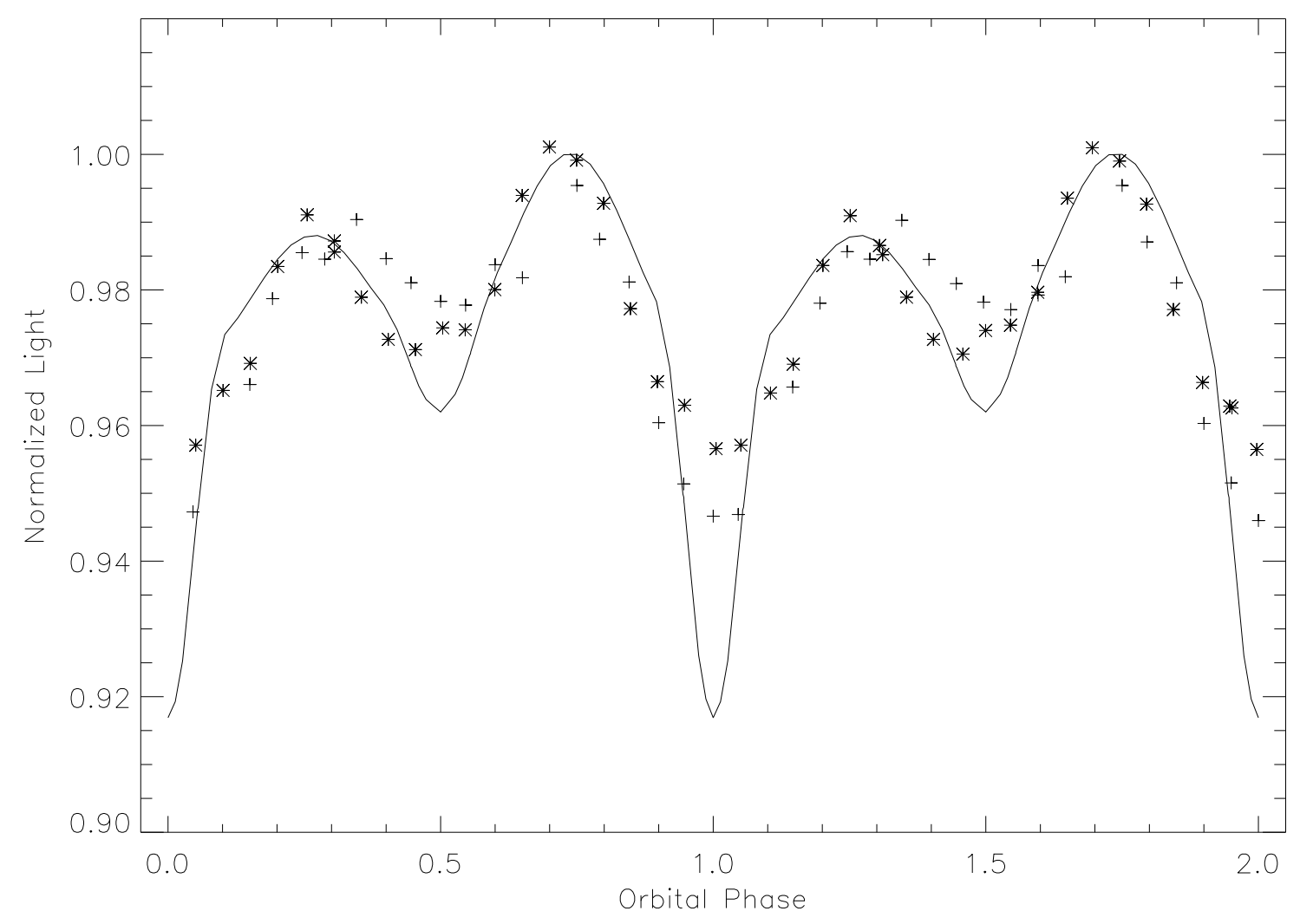

Fig. 7.- As in Figure 5 but for an orbital inclination of $60^{\circ}$. The light curve amplitude is too large. See the text for a discussion. 


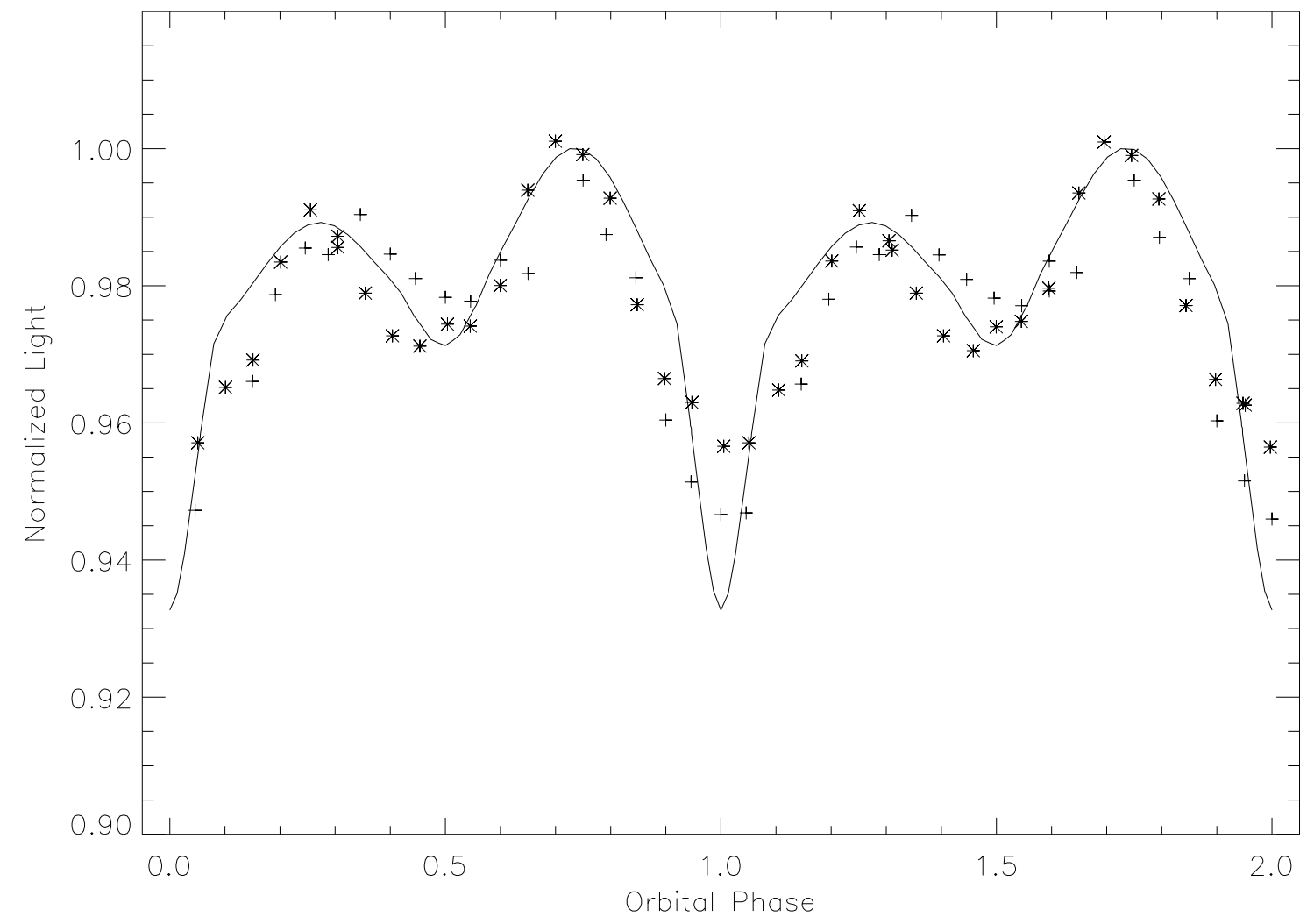

Fig. 8.- As in Figure 5 but for the final system parameters. See the text for a discussion. 


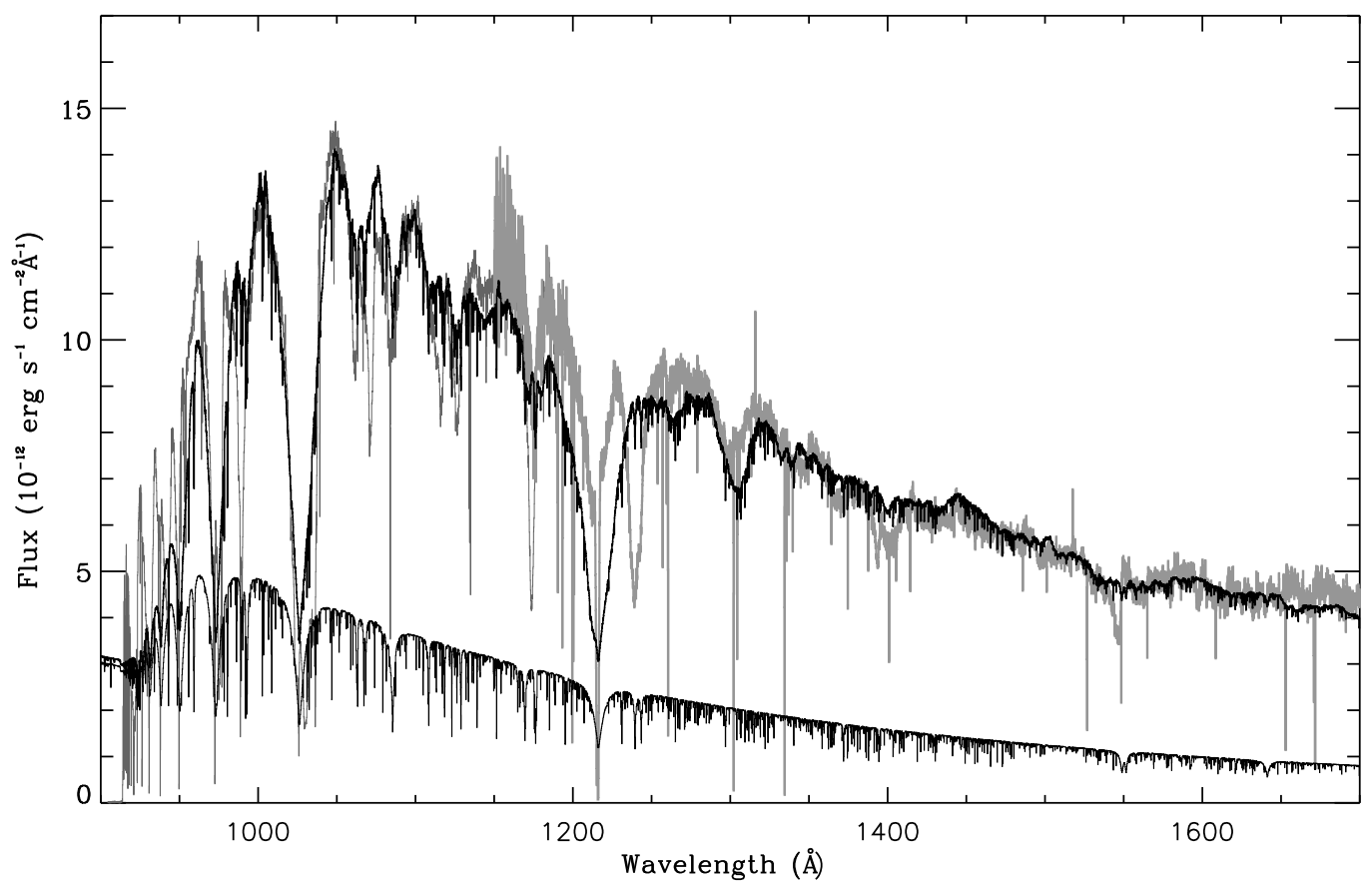

Fig. 9.- Synthetic system spectrum superposed on observed spectra. The light gray plot marks the FUSE spectrum; the darker gray plot marks the STIS spectrum. The contribution of a $60,000 \mathrm{~K} \mathrm{WD}$ is at the bottom. 


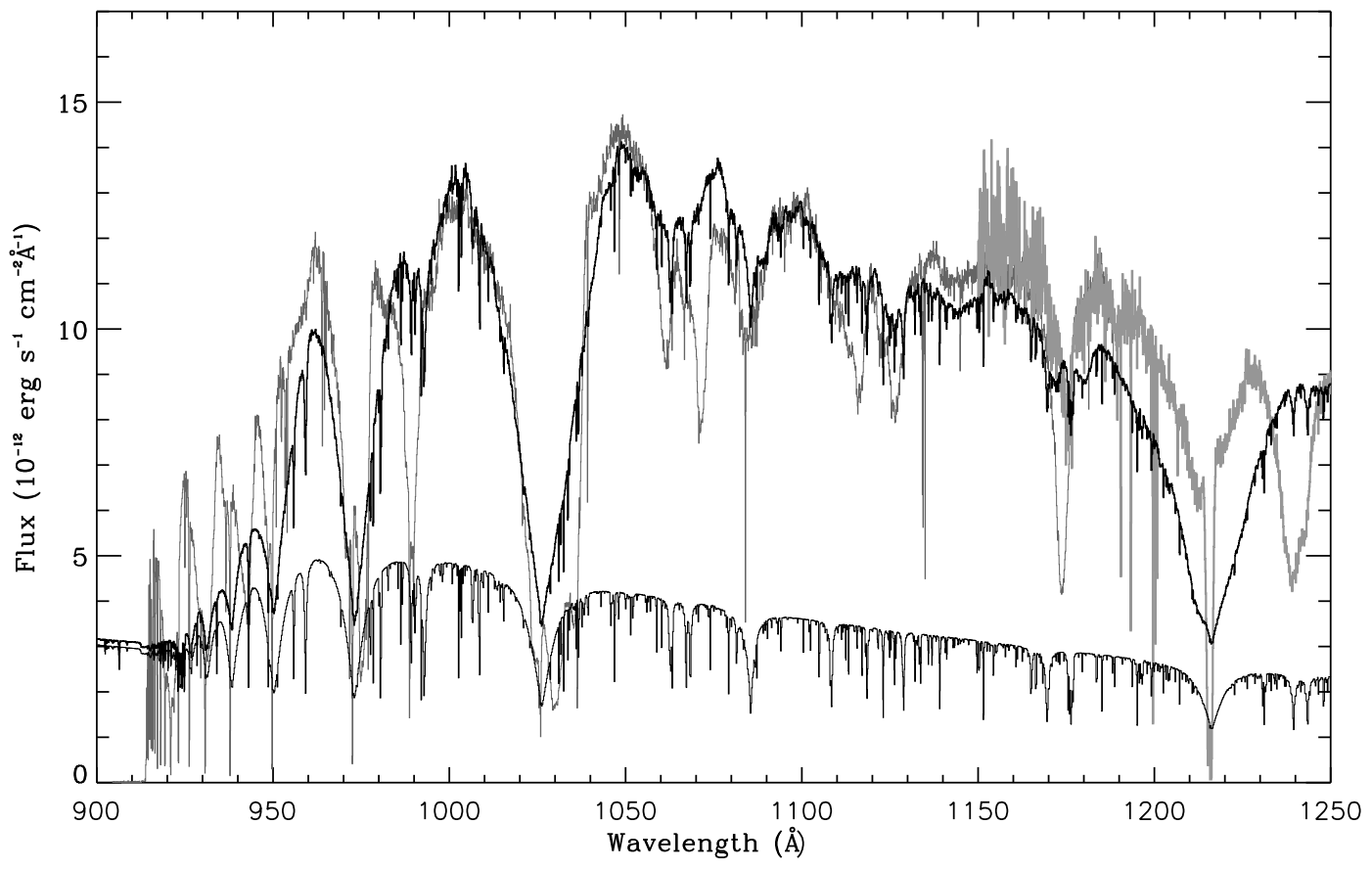

Fig. 10.- Detail of Figure 9. Note the strong and unmodeled wind lines. The contribution of a $60,000 \mathrm{~K}$ WD is at the bottom. 


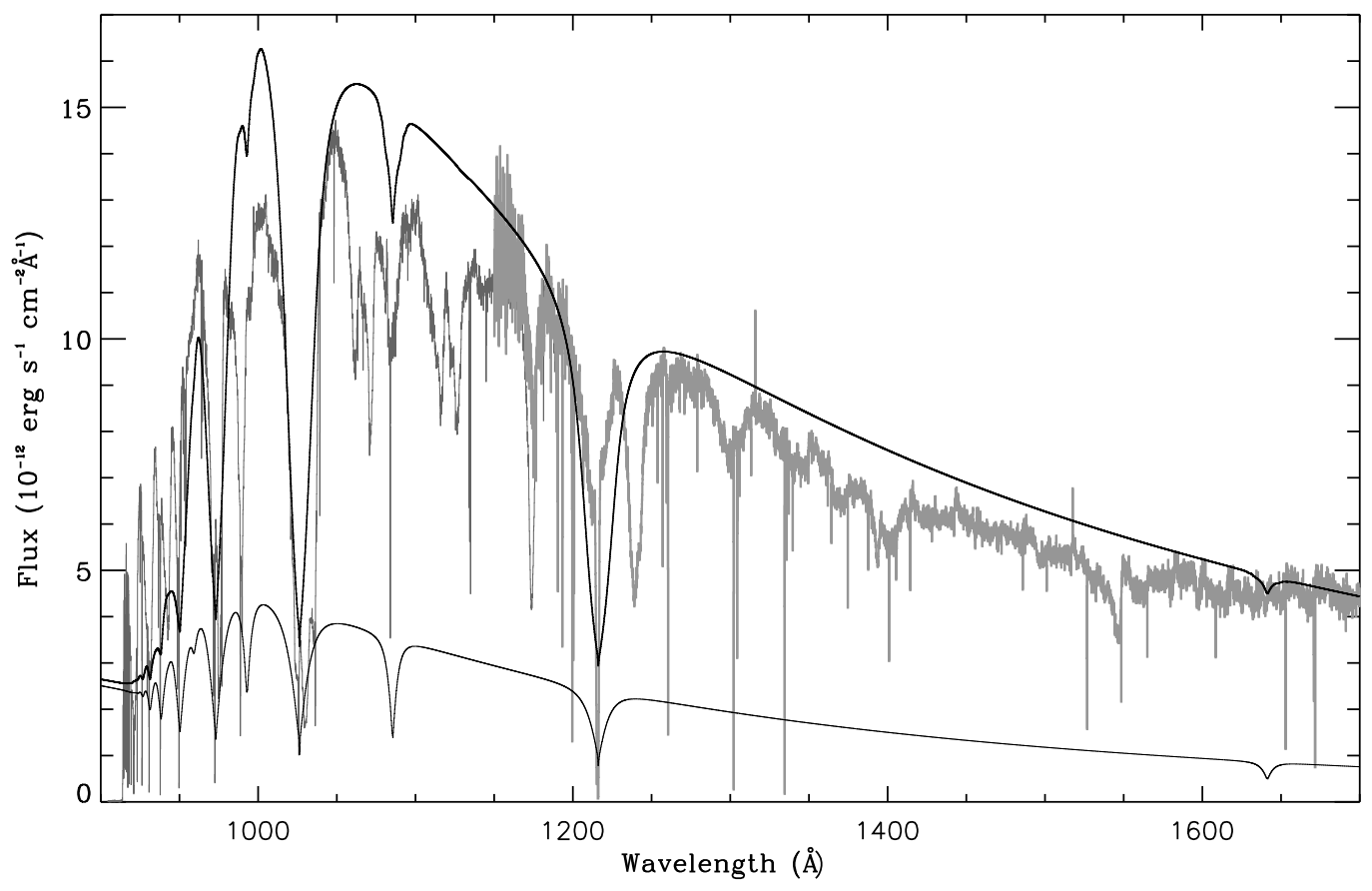

Fig. 11.- Synthetic system continuum spectrum plus H I and He II lines superposed on the observed spectra. Compare with Figure 9: Note that inclusion of line opacity (Figure 9) is necessary for a satisfactory fit. 


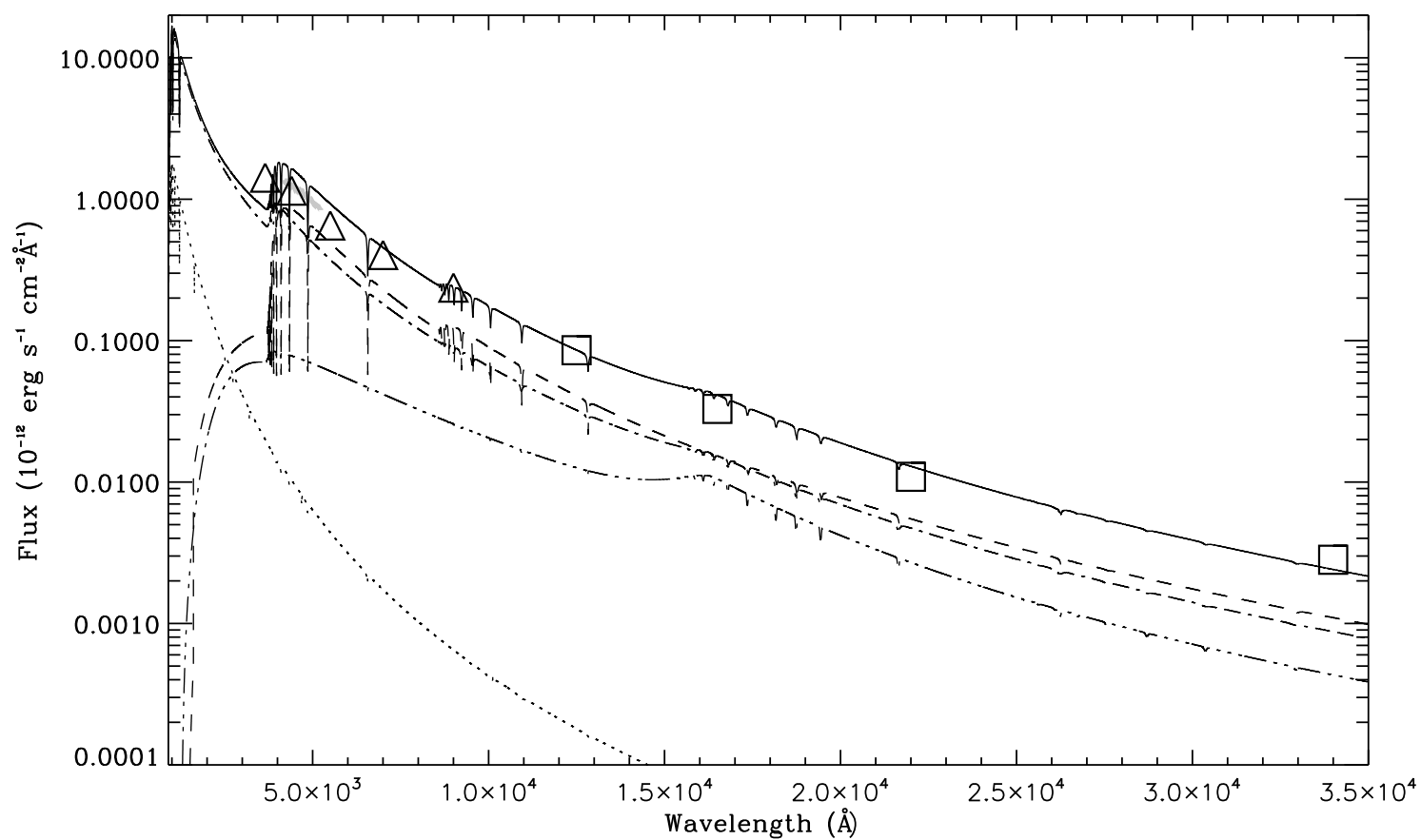

Fig. 12. - Plots of separate contributors to the system synthetic spectrum compared with photometric data. All synthetic spectra are theoretical continuum plus H I and He II lines. The continuous curve is the system synthetic spectrum; the dotted line is the WD; the dashed line is the secondary star; the dash dot line is the accretion disk face; the dash dot dot dot line is the accretion disk rim. The triangles represent UBVRI photometry by Garrison et al. (1984) and squares represent JHKL photometry by Haug (1988) and Wargau et al. (1984). 


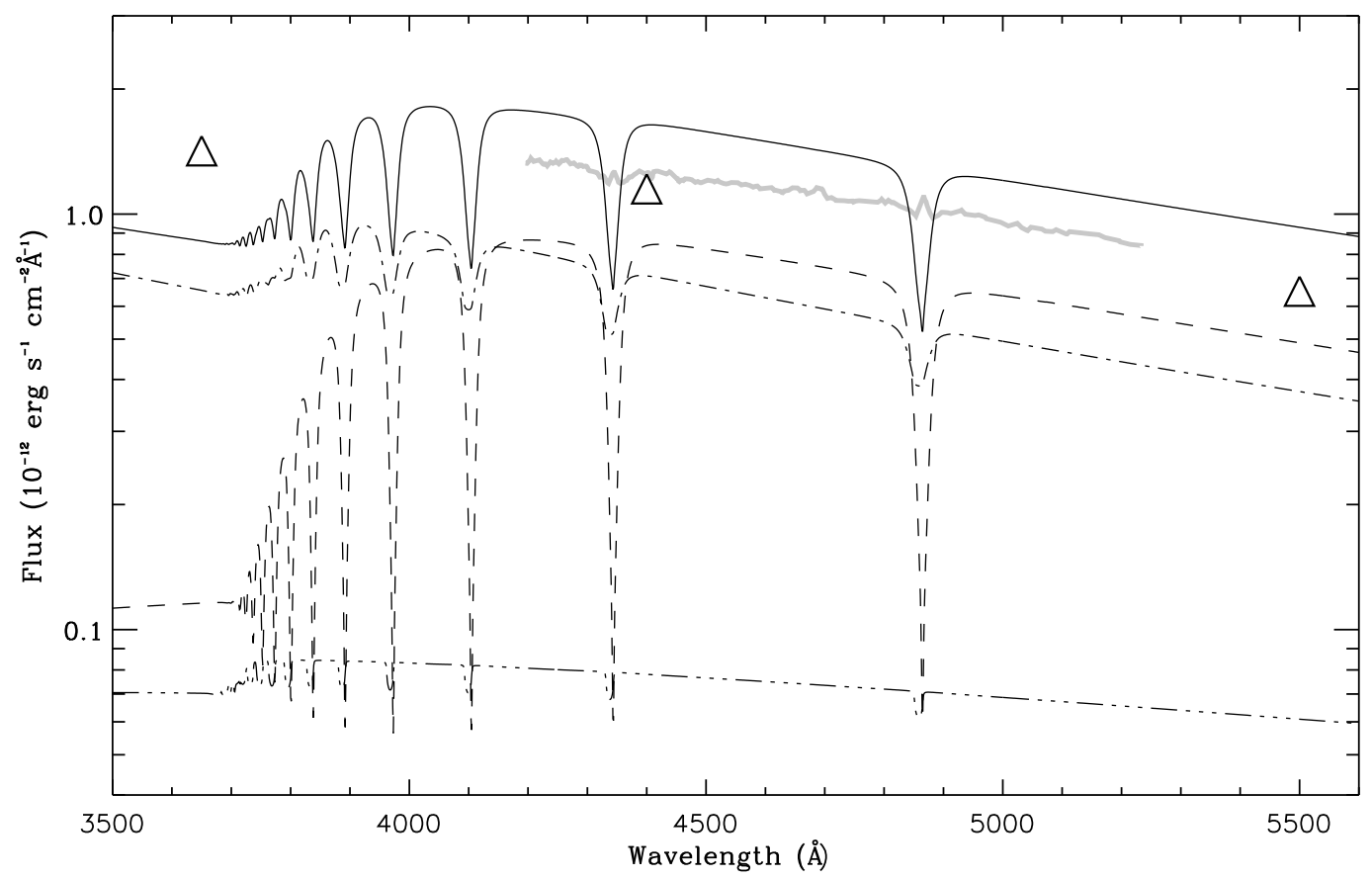

Fig. 13.- Detail of Figure 12. Based on the continuum spectra, the secondary star appears to be the major contributor to the line strengths of the system Balmer lines. The orbital phase is 0.25 , with the secondary star receding. Note the slight redward shift of the secondary star lines relative to the face and rim of the accretion disk lines. The lines of the latter two objects are rotationally broadened. The gray line, also appearing in Figure 12, is an optical spectrum by Wargau et al. (1983). 


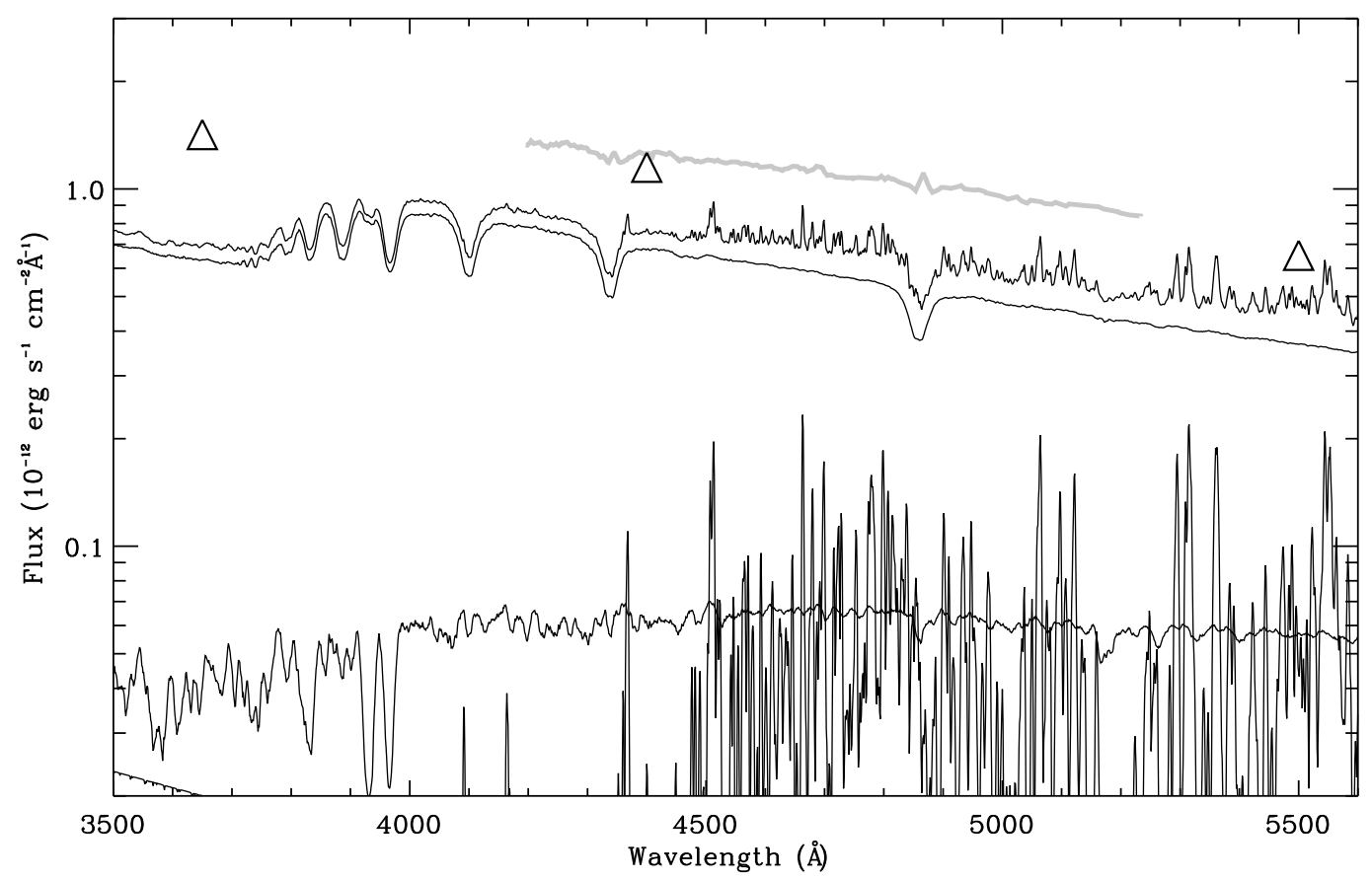

Fig. 14. - Line synthetic spectrum corresponding to Figure 13. Observational data as in Figure 12. The accretion disk face synthetic spectrum lies immediately below the system spectrum. The rim synthetic spectrum runs roughly horizontally across the plot, and the secondary star synthetic spectrum shows in the lower left corner and continues as the very jagged plot. This line spectrum shows that the accretion disk face actually is the major contributor to the Balmer lines. Compare with Figure 13; note the major change in the contribution of the secondary star. See the text for a discussion. 


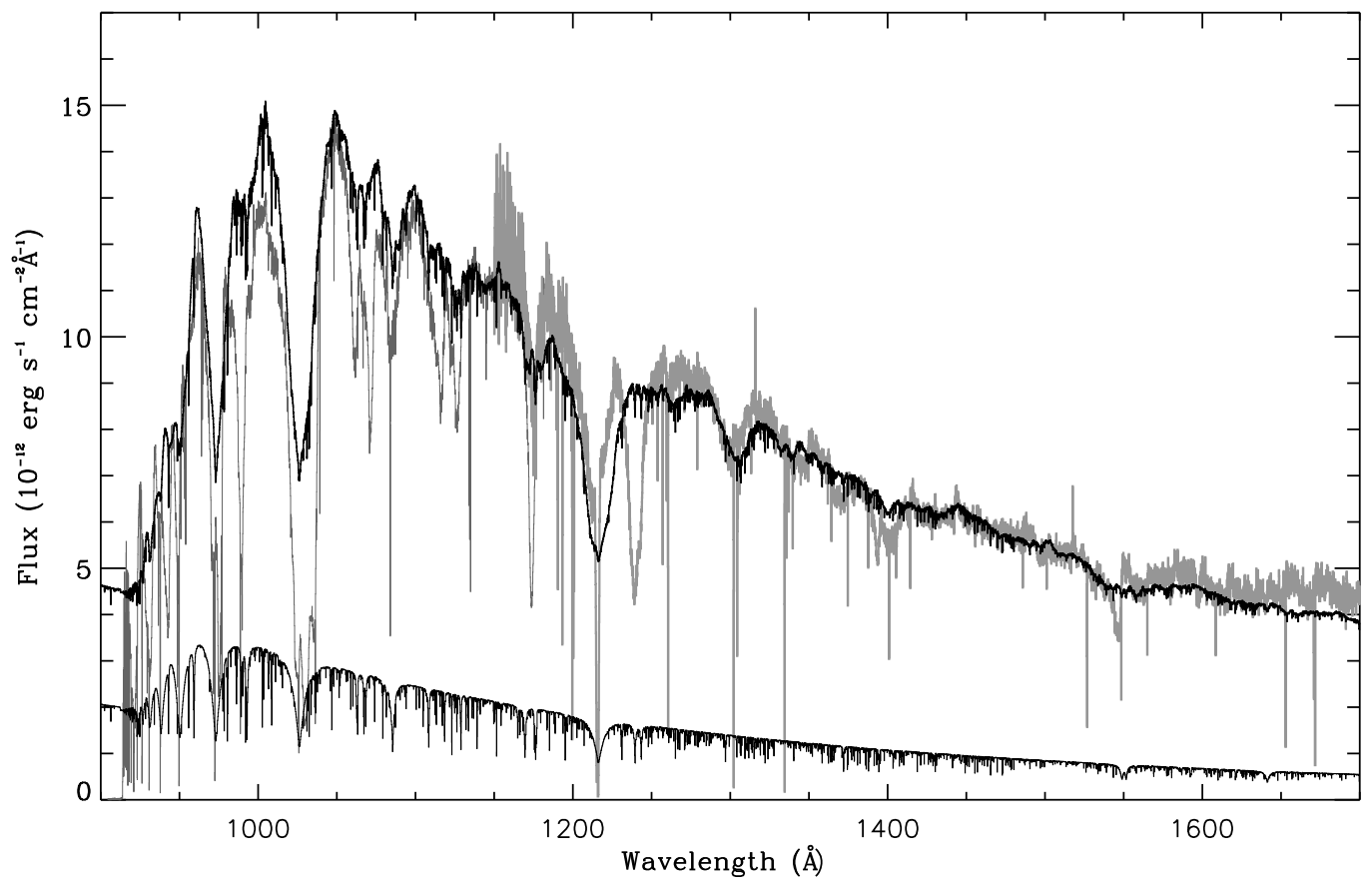

Fig. 15. - Standard model accretion disk for $\dot{M}=5.0 \times 10^{-9} M_{\odot} \mathrm{yr}^{-1}$ compared with FUSE and STIS spectra. See the text for a discussion. 